\title{
Charge-vibration interaction effects in normal-superconductor quantum dots
}

\author{
P. Stadler, ${ }^{1}$ W. Belzig, ${ }^{1}$ and G. Rastelli ${ }^{1,2}$ \\ ${ }^{1}$ Fachbereich Physik, Universität Konstanz, D-78457 Konstanz, Germany \\ ${ }^{2}$ Zukunftskolleg \& Fachbereich Physik, Universität Konstanz, D-78457, Konstanz, Germany
}

(Received 17 March 2017; published 24 July 2017)

\begin{abstract}
We study the quantum transport and the nonequilibrium vibrational states of a quantum dot embedded between a normal-conducting and a superconducting lead with the charge on the quantum dot linearly coupled to a harmonic oscillator of frequency $\omega$. To the leading order in the charge-vibration interaction, we calculate the current and the nonequilibrium phonon occupation by the Keldsyh Green's function technique. We analyze the inelastic, vibration-assisted tunneling processes in the regime $\omega<\Delta$, with the superconducting energy gap $\Delta$, and for sharp resonant transmission through the dot. When the energy $\varepsilon_{0}$ of the dot's level is close to the Fermi energy $\mu$, i.e., $\left|\varepsilon_{0}-\mu\right| \ll \Delta$, inelastic vibration-assisted Andreev reflections dominate up to voltage $e V \gtrsim \Delta$. The inelastic quasiparticle tunneling becomes the leading process when the dot's level is close to the superconducting gap $\left|\varepsilon_{0}-\mu\right| \sim \Delta \pm \omega$. In both cases, the inelastic tunneling processes appear as sharp and prominent peaks-not broadened by temperature-in the current-voltage characteristic and pave the way for inelastic spectroscopy of vibrational modes even at temperatures $T \gg \omega$. We also found that inelastic vibration-assisted Andreev reflections as well as quasiparticle tunneling induce a strong nonequilibrium state of the oscillator. In different ranges on the dot's level, we found that the current produces: (i) ground-state cooling of the oscillator with phonon occupation $n \ll 1$, (ii) accumulation of energy in the oscillator with $n \gg 1$, and (iii) a mechanical instability characterized by a negative damping coefficient. We show that ground-state cooling is achieved simultaneously for several modes of different frequencies. Finally, we discuss how the nonequilibrium vibrational state can be readily detected by the asymmetric behavior of the inelastic current peaks with respect to the gate voltage.
\end{abstract}

DOI: 10.1103/PhysRevB.96.045429

\section{INTRODUCTION}

Electronic transport through nanoscale devices is characterized by a variety of physical phenomena $[1,2]$. One of them is the interplay between quantum transport at the level of single electron and the mechanical motion of localized vibrations in various nanoscale devices. These include single-molecule junctions [3-13], suspended carbon nanotube quantum dots (CNT-QDs) [14-31], and several types of nanoelectromechanical systems (NEMS) [32-34] as single-electron transistors [35-39], superconducting single-electron transistors [40-45], single-electron shuttles [46-54], shuttling nanopilars $[55,56]$, and quantum dots in suspended nanostructures [57,58]. In such nanodevices the motion of the resonator can be not only detected but also manipulated via electron transport. At the same time, the mechanical motion strongly influences the transport itself.

For instance, in quantum dots, the interaction between the electrons and the harmonic vibrational modes gives rise to inelastic tunneling processes appearing when consecutive vibrational levels enter the bias window. Such an inelastic spectroscopy has been realized in experiments in single molecules [6,8,10-12] and in suspended CNT-QDs [16-20] at least for the high-frequency vibrational modes $\omega \gg T$ ( $\hbar=k_{B}=1$ ), viz. longitudinal or radial modes, but not for the low-frequency flexural modes. As the bias voltage increases, tunneling electrons can affect strongly the harmonic vibration leading to current-induced nonequilibrium vibrational states, in some cases up to the threshold of molecular dissociation [13].

More generally, charge-vibration interaction leads to a plethora of novel and unexpected behaviors of the nanoresonators. Electromechanical back-action effects-as oscillator's frequency shift and electromechanical dampinghave been reported in experiments for the flexural modes in suspended CNT-QDs [21,22,27,29], in quantum dots coupled to a piezoelectric nanoresonator [58] and in a dc-SQUID integrating a flexural resonator [59]. Further increasing the coupling strength, current suppression is expected due to the Franck-Condon blockade mechanism which was experimentally observed in CNT-QDs [19]. Mechanical bistabilities and blocked-current states are also theoretically predicted beyond a critical threshold of the charge-vibration coupling strength for low frequency (classical) nanoresonators [60-65]. Other theoretical studies reported negative electromechanical damping [66], self-sustained mechanical oscillations [67-70], and charge-induced nonlinear effects [27,71,72].

The majority of experimentally realized single-molecule junctions - as well as suspended CNT-QDs - can be properly modeled as single quantum dots [3]. Hence, the singleimpurity Holstein model [73-85] has become the paradigmatic model to discuss the effects of charge-vibration interaction in such systems. In its simple formulation, one assumes a linear coupling between the electron occupation on the dot and the oscillation's amplitude of one or more harmonic modes representing the local vibrations. This model has been theoretically investigated widely in literature with different variations, extensions, in different regimes and with various theoretical approaches [86-112], in particular using diagrammatic techniques [113-120], a resummation approach [121,122], and numerical and nonperturbative methods [123-127].

However, the above mentioned theoretical studies of the Holstein model focused on the case of a quantum dot in contact with two normal conducting leads (N-QD-N). Other theoretical works investigated the problem of quantum dots 
(a)

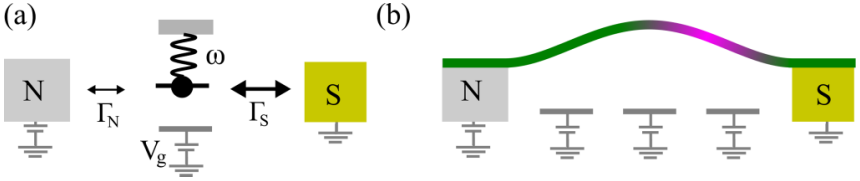

FIG. 1. (a) Sketch of a quantum dot coupled to an oscillator of frequency $\omega$. The dot's energy level is controlled by the gate voltage $V_{g}$. The quantum dot is in contact with a normal conductor and a superconductor with gap $\Delta$, and the tunneling rates are $\Gamma_{N}$ and $\Gamma_{S}$, respectively. (b) Suspended carbon nanotube quantum dot with the dot's charge coupled to the vibrational flexural modes, in the regime of $\Gamma_{N} \ll \Gamma_{S}$ with multigates, see Ref. [29].

coupled to the local vibrations and between two superconductors (S-QD-S) as a Josephson dot coupled to several bosonic modes [128], a suspended carbon nanotube acting as nanomechanical resonator [67,129-134], or a molecular Josephson junction model [135]. The latter was used to explain the resonance peaks experimentally observed in vibrating $\mathrm{Nb}$ nanowires $[136,137]$.

On the contrary, the hybrid case of a normal-dotsuperconductor (N-QD-S) coupled to the bosonic modes-as photons [138,139] or phonons [140-142]-has been less studied in the literature and is theoretically unexplored for the nonequilibrium regime of the oscillator. In this system, one expects that the role of the superconducting lead is unessential for $\Delta \ll \omega$ ( $\Delta$ the superconducting gap) as the vibrational frequency $\omega$ sets the energy scale of the inelastic tunneling of electrons. The interesting and relevant regime is thus expected for $\Delta \sim \omega$ or $\Delta>\omega$, which corresponds to a demanding condition in single-molecule junctions characterized by typical high-frequency vibrations due to the low mass of the molecules, see Ref. [2]. On the other hand, this regime can occur naturally in suspended CNT-QDs, see Fig. 1. Moreover, CNT-QDs can be inserted between different types of nanocontacts, as a normal metal and a superconductor [143-147].

In a previous recent work [148] we explored the NQD-S system with charge-vibration interaction in the deep subgap regime corresponding to a gap much larger than all energy scales (viz. $\Delta \rightarrow \infty$ ). Our main finding was that the vibrational modes can be cooled to the ground state by inelastic vibration-assisted Andreev reflections with phonon number occupation $n \ll 1$ up to the back-action limit dictated by the shot noise and given by $n_{B A} \sim\left(\Gamma_{N} / \omega\right)^{2}\left(\Gamma_{N}\right.$ the tunneling rate with the normal lead). We also showed that such a method of cooling is exploitable even for several mechanical modes. Ground-state cooling by electron transport in suspended CNT-QDs appears as a promising strategy to achieve the quantum regime of low-frequency flexural modes $\omega \ll T$. It has been the subject of a notable research activity during the past years [148-157]. We recall that cooling by electron transport has been experimentally demonstrated in nanomechanical beams integrating normal metal-insulatorsuperconductor tunnel junctions $[158,159]$.

The objective of this paper is to discuss the system $\mathrm{N}-\mathrm{QD}-\mathrm{S}$ with charge-vibration interaction at large but finite superconducting gap $\Delta$. We aim at demonstrating that the $\mathrm{N}-\mathrm{QD}-\mathrm{S}$ is indeed of particular interest as: (i) it is characterized (a)

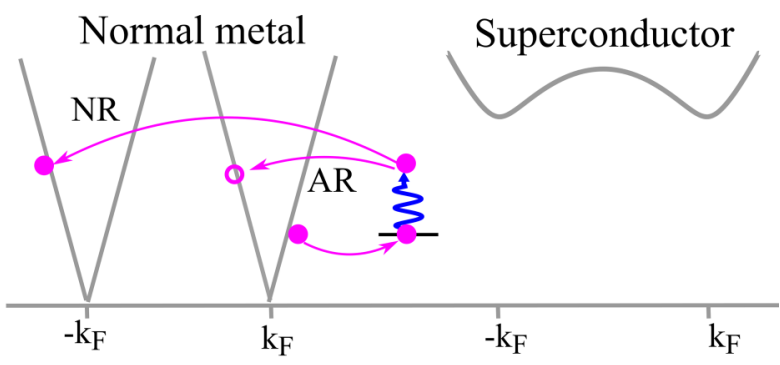

(b)

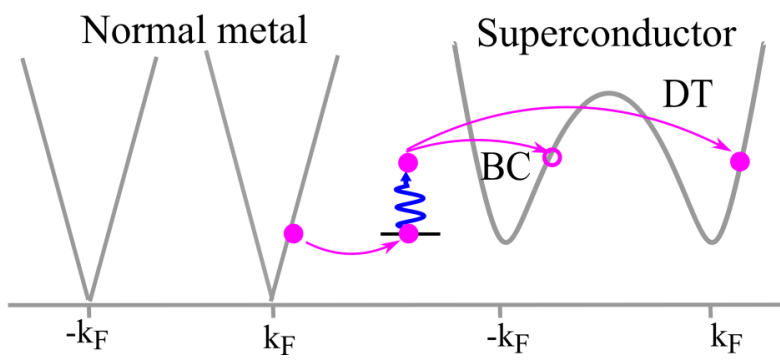

FIG. 2. Examples of inelastic vibration-assisted tunneling processes occurring in a N-QD-S system with charge-vibration interaction. The electron(hole)-like excitations are sketched as filled (empty) circles, respectively, and the solid lines show the energy spectrum around the Fermi momentum $k_{F}$. An electronlike excitation incoming from the normal metal tunnels to the quantum dot and absorbs a phonon with frequency $\omega$ (wiggled vertical arrow). (a) Below the gap an incoming quasiparticle on the normal metal can be either Andreev reflected (AR) or normal reflected (NR). (b) Close to the gap, in addition to AR and NR, the incoming quasiparticle can inelastically tunnel toward the superconducting lead by direct tunneling (DT) or branch crossing (BC).

by an intriguing behavior of the inelastic transport ruled by inelastic Andreev reflections as well as by inelastic quasiparticle tunneling [see Fig. 2], (ii) it opens the route to inelastic spectroscopy of the low-frequency modes as the flexural ones which cannot be resolved using normal leads at temperature $T \gg \omega$, and (iii) it allows strong manipulation of the vibrational states via electron transport ranging from ground-state cooling up to a mechanical instability. Finally, such a system offers intrinsically the possibility to read out the equilibrium or the nonequilibrium state of the vibration directly by dc-current measurements.

We found that the results in the subgap regime, at large but finite superconducting gap, are indistinguishable from our previous results obtained for $\Delta \rightarrow \infty$ in Ref. [148] pointing out the robustness of our previous approximation. In comparison to our previous work, the genuine novel physics of this work is the study of the inelastic tunneling of quasiparticles that also affects substantially the vibrational phonon occupation for gate voltage close to the superconducting gap. As result, we obtain a more rich behavior shown, for instance, in Fig. 6(a). We further report analytic expressions for the phonon occupation and the current at finite gap and for arbitrary bias and gate voltages.

The paper is structured as follows. In Sec. II we introduce the Holstein model of a N-QD-S with charge-vibration interaction and discuss the range of validity of our model and of the perturbative diagrammatic expansion. In Sec. III we discuss 
the results for the electromechanical damping $\gamma$ showing that it is related to several microscopic processes, in particular the inelastic Andreev reflection and inelastic quasiparticle tunneling between the normal to the superconducting lead [see Fig. 2].

The phonon number occupation is discussed in Sec. IV. In Sec. IV A we discuss the phonon occupation for a single vibrational mode. Remarkably, in the limit $\omega \ll \Delta$, there is a separation for the dot's energy ranges where the inelastic Andreev reflections dominates over the inelastic quasiparticles tunneling or vice versa. Both kinds of processes can yield ground-state cooling of the oscillator with phonon occupation $n \ll 1$, an accumulation of energy in the oscillator with $n \gg 1$ and a mechanical instability signaled by the negativity of the electromechanical damping. This region, as well as the region of $n \gg 1$, are beyond our perturbative approach and the validity of harmonicity of the resonator. In the Sec. IV B we generalize the discussion taking into account many vibrational modes showing that ground-state cooling can be achieved simultaneously for several $\mathrm{N}$ modes of different frequencies $\omega_{n}$ via inelastic quasiparticle tunneling.

In Sec. $\mathrm{V}$ we show our results for the current. We discuss mainly the inelastic correction to the current and show how the nonequilibrium state of the vibration can be properly read out by the asymmetric behavior of inelastic current peaks with respect to the gate voltage. Section VI contains concluding remarks.

\section{MODEL AND APPROXIMATION}

In this section, we discuss a model of a quantum dot suspended between a normal-conducting and a superconducting contact which can be realized, e.g., with a suspended CNT-QD (see Fig. 1). We consider the electrons on the quantum dot occupying a spin-degenerate state with energy $\varepsilon_{0}$ and the annihilation and creation operators $\hat{d}_{\sigma}$ and $\hat{d}_{\sigma}^{\dagger}$ with spin $\sigma$ on the quantum dot. The occupation number reads $\hat{n}_{d}=\hat{d}_{\uparrow}^{\dagger} \hat{d}_{\uparrow}+\hat{d}_{\downarrow}^{\dagger} \hat{d}_{\downarrow}$. A single harmonic mode of the resonator with frequency $\omega$ has the bosonic annihilation and creation operators $\hat{b}$ and $\hat{b}^{\dagger}$. The electrostatic force between the electrons and a gate voltage induces a coupling between the charge and the deflection of the resonator with coupling strength $\lambda$. The full Hamiltonian is given by

$$
\hat{H}=\hat{H}_{N}+\hat{H}_{S}+\hat{H}_{T}+\varepsilon_{0} \hat{n}_{d}+\lambda \hat{n}_{d}\left(\hat{b}^{\dagger}+\hat{b}\right)+\omega \hat{b}^{\dagger} \hat{b},
$$

with the last two terms describing the charge-vibration coupling and the resonator. The Hamiltonians of the normal and the superconducting leads are given by $\hat{H}_{N}=\sum_{k \sigma} \xi_{N, k} \hat{c}_{k \sigma}^{\dagger} \hat{c}_{k \sigma}$ and $\hat{H}_{S}=\sum_{k \sigma}\left(\xi_{S, k} \hat{a}_{k \sigma}^{\dagger} \hat{a}_{k \sigma}+\Delta \hat{a}_{-k \downarrow}^{\dagger} \hat{a}_{k \uparrow}^{\dagger}+\Delta \hat{a}_{k \uparrow} \hat{a}_{-k \downarrow}\right)$ with the real pairing potential $\Delta$ (superconducting gap) and the energy referring to the chemical potential $\xi_{\alpha, k}=\varepsilon_{k}-\mu_{\alpha}$ with $\alpha=(N, S)$. The annihilation operators with spin $\sigma$ and momentum $k$ are given by $\hat{a}_{k \sigma}$ for the superconducting and $\hat{c}_{k \sigma}$ for the normal-conducting lead. The tunneling Hamiltonian is $\hat{H}_{T}=\sum_{k \sigma}\left(t_{N} \hat{c}_{k \sigma}^{\dagger} \hat{d}_{\sigma}+t_{S} \hat{a}_{k \sigma}^{\dagger} \hat{d}_{\sigma}+\right.$ H.c. $)$ with the tunneling energies between the leads and the dot $t_{N}$ and $t_{S}$. In the rest of our analysis we assume the wide-band approximation and write the tunnel couplings between the quantum dot with the normal contact and with the superconducting contact as
$\Gamma_{N}=\pi\left|t_{N}\right|^{2} \rho_{N}$ and $\Gamma_{S}=\pi\left|t_{S}\right|^{2} \rho_{S}$, respectively. The electronic density of states at the Fermi level of the normal lead and of the superconducting lead in the normal (nonsuperconducting) phase are denoted by $\rho_{N}$ and $\rho_{S}$.

Before we conclude this section, we discuss the role of the Coulomb interaction in the dot. In the past, the Anderson model with local Hubbard repulsion $U$ in a N-QD-S system without charge vibration interaction was studied to understand the competition between superconducting pairing, Coulomb repulsion, and Kondo correlations [160-165]. Analytic methods were restricted to truncated approximated expansions in the regime $U \rightarrow \infty$ and a finite superconducting gap [160-162]. An interpolation approach was used in Ref. [163] for a wide range of the ratio of $U / \Gamma_{S}$, showing that, for $U / \Gamma_{S}<1$, superconducting pairing dominates the behavior of the quantum dot. Tanaka et al. [164] employed a numerical renormalization group method in the limit $\Delta \rightarrow \infty$ which allows us to map the problem to the case of a QD with a local pairing amplitude $\Delta_{D}=\Gamma_{S}$. Reference [164]-limited in the linear transport regime-points out that the effect of the interaction is to renormalize the coupling $\Gamma_{S}$ and the dot level $\varepsilon_{0}$ in the regime $U \lesssim \Gamma_{S}$. A similar approach was employed in S-QD-S [166,167] and N-QD-S [168,169] junctions which focused on the regime $\Gamma_{S} \gg \Gamma_{N}$ and in the large gap limit to discuss the effective Hamiltonian for the proximized quantum dot whose behavior is ruled by the ration $U / \Gamma_{S}$. This approach predicts essentially that, when $\Gamma_{S} \gg \Gamma_{N}$ it is still possible to establish a BCS-like state in a single-level quantum dot even in the presence of Coulomb repulsion, i.e., $U / \Gamma_{S}<1$. We notice that, in our problem and large gap, we recover the same effective Hamiltonian with $U=0$ [148], viz. $\hat{H}_{d S}=\varepsilon_{0} \sum_{\sigma} \hat{d}_{\sigma}^{\dagger} \hat{d}_{\sigma}-\Gamma_{S}\left(\hat{d}_{\downarrow} \hat{d}_{\uparrow}+\right.$ H.c. $)$.

Tackling the problem of systems with Coulomb interaction and charge vibration shows a formidable task beyond the scope of this work. Here we assume the tunneling rate with the normal lead $\Gamma_{N}$ as the lowest energy involved in the problem and the condition for the energy scales $U \lesssim \Gamma_{S}<\Delta$ which sets a conservative estimation for the range of validity of our approach. Finally, we remark that experiments with $U \lesssim \Delta$ as in Refs. [145,146,170] focused on devices with large asymmetry $\Gamma_{S} \gg \Gamma_{N}$ the normal lead basically acts as a spectroscopic probe of the Andreev spectra. In some ranges of parameters, the experimental results have been qualitatively explained in terms of a noninteracting model, viz. the Kondo effects and correlations seems suppressed and play no role.

\section{A. Exact results for the quantum dot for $\lambda=0$}

In this subsection we recall the exact results for the electronic Green's function on the dot without charge-vibration interaction and the behavior of the density of state of the quantum dot $[171,172]$. These Green's functions are the building block by which we can express the electromechanical damping, the phonon occupation, the zero-order current, and the current corrections in the presence of the charge-vibration interaction.

In Keldysh space, we write the electronic Green's function as

$$
\check{G}\left(t, t^{\prime}\right)=\left(\begin{array}{cc}
\hat{G}^{R}\left(t, t^{\prime}\right) & \hat{G}^{K}\left(t, t^{\prime}\right) \\
0 & \hat{G}^{A}\left(t, t^{\prime}\right)
\end{array}\right),
$$


with the retarded (R), advanced (A) and Keldysh (K) Green's functions defined as $\hat{G}^{R}\left(t, t^{\prime}\right)=-i \theta\left(t-t^{\prime}\right)\left\langle\left\{\Psi_{d}(t), \Psi_{d}^{\dagger}\left(t^{\prime}\right)\right\}\right\rangle$, $\hat{G}^{A}\left(t, t^{\prime}\right)=i \theta\left(t^{\prime}-t\right)\left\langle\left\{\Psi_{d}(t), \Psi_{d}^{\dagger}\left(t^{\prime}\right)\right\}\right\rangle, \quad$ and $\hat{G}^{K}\left(t, t^{\prime}\right)=$ $-i\left\langle\left[\Psi_{d}(t), \Psi_{d}^{\dagger}\left(t^{\prime}\right)\right]\right\rangle$. Here, we introduced the spinor in electron-hole space as $\Psi_{d}^{\dagger}(t)=\left(d_{\uparrow}^{\dagger}(t) d_{\downarrow}(t)\right)$ acting in the Nambu space and denote the commutator and anticommutator with $[$,$] and \{$,$\} , respectively. Notice the notation { }^{`}$ for the matrix defined on the Keldysh contour space and`for the matrix defined on the Nambu space. The notation of the Green's function in Nambu space follows Ref. [173]. The Dyson equation for the Green's function on the dot is $\check{G}(\varepsilon)=$ $\check{g}(\varepsilon)+\check{g}(\varepsilon)\left[\check{\Sigma}_{N}(\varepsilon)+\check{\Sigma}_{S}(\varepsilon)\right] \breve{G}(\varepsilon)$, with the unperturbed Green's function of the dot $\breve{g}(\varepsilon)$ and the self-energies $\check{\Sigma}_{\alpha}(\varepsilon)$ of the normal and superconducting lead $[\alpha=(N, S)]$.

In the following, we apply the voltage on the normalconducting lead $\xi_{N, k}=\varepsilon_{k}-\mu-e V, \xi_{S, k}=\varepsilon_{k}-\mu$ and let $\mu=0$. Using the wide-band approximation, the retarded and Keldysh self-energies of the normal metal are $\hat{\Sigma}_{N}^{R}=-i \Gamma_{N}$ and $\hat{\Sigma}_{N}^{K}(\varepsilon)=-2 i \Gamma_{N}[1-2 \hat{F}(\varepsilon)]$ with the matrix function

$$
\hat{F}(\varepsilon)=\left(\begin{array}{cc}
f_{+}(\varepsilon) & 0 \\
0 & f_{-}(\varepsilon)
\end{array}\right),
$$

in which we define the two functions $f_{v}(\varepsilon)=\{1+\exp [(\varepsilon-$ $v e V) / T]\}^{-1}$ with the index $v= \pm=(e, h)$ to account for the sign of the charge for the electrons and holes, respectively. The retarded and Keldysh self-energies of the superconductor are

$$
\hat{\Sigma}_{S}^{R}(\varepsilon)=-\frac{\Gamma_{S}}{\Omega(\varepsilon)}\left(\begin{array}{cc}
\varepsilon+i \eta & -\Delta \\
-\Delta & \varepsilon+i \eta
\end{array}\right)
$$

and $\hat{\Sigma}_{S}^{K}(\varepsilon)=\left[1-2 f_{0}(\varepsilon)\right]\left[\hat{\Sigma}_{S}^{R}(\varepsilon)-\hat{\Sigma}_{S}^{A}(\varepsilon)\right]$ with $\Omega(\varepsilon)=$ $\sqrt{\Delta^{2}-(\varepsilon+i \eta)^{2}}$, an infinitesimal small real part $\eta$ and the Fermi function of the superconducting lead $f_{0}(\varepsilon)(v=0)$. Solving the Dyson equation, we obtain the retarded Green's function of the dot

$$
\hat{G}^{R}(\varepsilon)=\left(\begin{array}{cc}
G_{+}(\varepsilon) & F(\varepsilon) \\
F(\varepsilon) & G_{-}(\varepsilon)
\end{array}\right),
$$

with

$$
\begin{gathered}
G_{ \pm}(\varepsilon)=-\frac{1}{D(\varepsilon)}\left[\varepsilon \pm \varepsilon_{0}+i \Gamma_{N}+\varepsilon \Gamma_{S} / \Omega(\varepsilon)\right], \\
F(\varepsilon)=\frac{1}{D(\varepsilon)}\left[\Delta \Gamma_{S} / \Omega(\varepsilon)\right]
\end{gathered}
$$

and

$$
\begin{aligned}
D(\varepsilon)= & \frac{\Delta^{2} \Gamma_{S}^{2}}{\Omega^{2}(\varepsilon)}-\left[\varepsilon+\varepsilon_{0}+i \Gamma_{N}+\frac{\varepsilon \Gamma_{S}}{\Omega(\varepsilon)}\right] \\
& \times\left[\varepsilon-\varepsilon_{0}+i \Gamma_{N}+\frac{\varepsilon \Gamma_{S}}{\Omega(\varepsilon)}\right] .
\end{aligned}
$$

The Keldysh Green's function of the dot is obtained from the Dyson equation and is given by $\hat{G}^{K}(\varepsilon)=\hat{G}^{R}(\varepsilon)\left(\hat{\Sigma}_{N}^{K}(\varepsilon)+\right.$ $\left.\hat{\Sigma}_{S}^{K}(\varepsilon)\right) \hat{G}^{A}(\varepsilon)$.

The density of states $\rho_{d}(\varepsilon)$ on the quantum dot is calculated by

$$
\rho_{d}(\varepsilon)=-\operatorname{Im} \operatorname{Tr}\left[\hat{G}^{R}(\varepsilon)\right]
$$
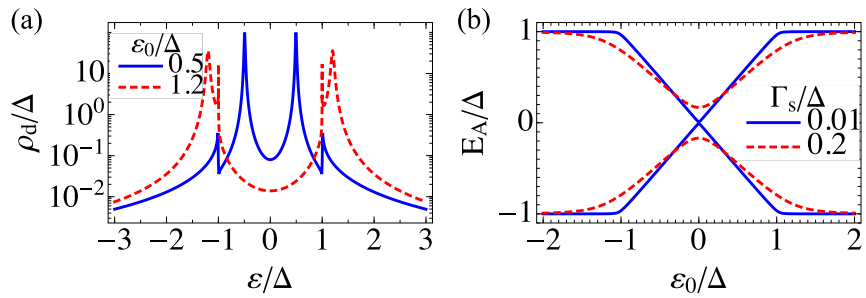

FIG. 3. (a) Density of states of the quantum dot $\rho_{d}(\varepsilon)$ as a function of $\varepsilon$ at $\Gamma_{N}=\Gamma_{S}=0.1 \Delta$ and for different bare dot's level $\varepsilon_{0}$. (b) Andreev levels $E_{A}$ as a function of $\varepsilon_{0}$ for $\Gamma_{N}=0.01 \Delta$ and different $\Gamma_{S}$. For $\varepsilon_{0}>\Delta$, the Andreev levels are very close to the gap edge and, additionally, a resonant peak occurs at $\varepsilon_{0}$.

with the retarded Green's function given by Eq. (5). An example of the density of states as a function of energy is shown in Fig. 3(a). For $|\varepsilon| \gg \Delta, \rho_{d}(\varepsilon)$ shows a similar behavior as for a quantum dot coupled to two normal leads. At low energy, the superconducting correlation penetrates on the quantum dot and leads to a suppression of the density of states and to the formation of Andreev levels pinned inside the superconducting gap and signaled by the two peaks. These Andreev levels $E_{A}$ have an energy dependence given by the zeroing of $D(\varepsilon)$ in Eq. (8) [see Fig. 3(b)]. For large superconducting gap, the Andreev levels are approximated by $E_{A} \simeq \pm \sqrt{\varepsilon_{0}^{2}+\Gamma_{S}^{2}}$ valid for $\left|E_{A}\right| \ll \Delta$.

\section{B. Phonon Green's function with charge-vibration interaction}

In this section we discuss the phonon Green's function for a single harmonic oscillator coupled to the N-QD-S system. We use the perturbative expansion to calculate the phonon self-energy $\breve{\Pi}(\varepsilon)$ to lowest order in the chargevibration coupling $[83,117]$. In this way, we can obtain the electromechanical damping and the nonequilibrium phonon occupation. Analytic formulas are possible in the limit of small damping $\gamma \ll \omega$ and a high quality factor of the resonator. Such an approach is equivalent to solve the quantum Langevin equation for the harmonic oscillator assuming as input the bare quantum noise correlators of the dot's charge in the N-QD-S without charge-vibration interaction [174] and using the Markovian approximation. The latter approximation holds in our case since the typical frequency scale $\delta \omega$ of the general electromechanical damping and of the noise scales as $\delta \omega \sim \Gamma_{N}$, and this quantity is still larger than the linewidth of the oscillator's susceptibility that scales as $\sim \lambda^{2} \Gamma_{N} / \omega^{2}$ in the regime $\Gamma_{N}<\omega$.

We introduce the symmetrized bosonic operator $\hat{A}(t)=$ $\hat{b}^{\dagger}(t)+\hat{b}(t)$, the contour ordering operator $T_{c}$ on the Keldysh contour and define the phonon Green's function as $D\left(\tau, \tau^{\prime}\right)=$ $-i\left\langle T_{c} \hat{A}(\tau) \hat{A}^{\dagger}\left(\tau^{\prime}\right)\right\rangle$ with the time variable $\tau$ on the Keldysh contour. Then, we transform the contour variable $\tau$ to the real time and use the Larkin-Ovchinnikov rotation to write the Dyson equation in the triangular form

$$
\check{D}\left(t, t^{\prime}\right)=\left(\begin{array}{cc}
D^{R}\left(t, t^{\prime}\right) & D^{K}\left(t, t^{\prime}\right) \\
0 & D^{A}\left(t, t^{\prime}\right)
\end{array}\right),
$$

with the phonon Green's functions $D^{R}\left(t, t^{\prime}\right)=-i \theta(t-$ $\left.t^{\prime}\right)\left\langle\left[\hat{A}(t), \hat{A}\left(t^{\prime}\right)\right]\right\rangle, \quad D^{A}\left(t, t^{\prime}\right)=i \theta\left(t^{\prime}-t\right)\left\langle\left[\hat{A}(t), \hat{A}\left(t^{\prime}\right)\right]\right\rangle, \quad$ and 
$D^{K}\left(t, t^{\prime}\right)=-i\left\langle\left\{\hat{A}\left(t^{\prime}\right), \hat{A}(t)\right\}\right\rangle$. Finally, we obtain the Dyson equation in Fourier space as $\check{D}(\varepsilon)=\check{D}_{0}(\varepsilon)+$ $\check{D}_{0}(\varepsilon) \check{\Pi}(\varepsilon) \check{D}_{0}(\varepsilon)$. The bare phonon Green's functions in $\check{D}_{0}(\varepsilon)$ are given by $D_{0}^{R, A}(\varepsilon)=2 \omega /\left[(\varepsilon \pm i \eta)^{2}-\omega^{2}\right]$ and $D_{0}^{K}(\varepsilon)=-2 \pi i[\delta(\varepsilon-\omega)+\delta(\varepsilon+\omega)] \operatorname{coth}[\omega /(2 T)]$. The polarization $\check{\Pi}(\varepsilon)$ corresponds to the phonon self-energy (bubble diagram) to the leading order in the electron-vibration interaction $\sim \lambda^{2}$ between the harmonic oscillator and the electrons. These self-energies are given by

$$
\begin{aligned}
\Pi^{R, A}(\varepsilon)= & i \frac{\lambda^{2}}{2} \int \frac{d \varepsilon^{\prime}}{2 \pi} \operatorname{Tr}\left[\hat{\tau}_{z} \hat{G}^{K}\left(\varepsilon^{\prime}\right) \hat{\tau}_{z} \hat{G}^{A, R}\left(\varepsilon^{\prime}-\varepsilon\right)\right. \\
& \left.+\hat{\tau}_{z} \hat{G}^{R, A}\left(\varepsilon^{\prime}\right) \hat{\tau}_{z} \hat{G}^{K}\left(\varepsilon^{\prime}-\varepsilon\right)\right],
\end{aligned}
$$

and

$$
\begin{aligned}
\Pi^{K}(\varepsilon)= & i \frac{\lambda^{2}}{2} \int \frac{d \varepsilon^{\prime}}{2 \pi} \operatorname{Tr}\left[\hat{\tau}_{z} \hat{G}^{K}\left(\varepsilon^{\prime}\right) \hat{\tau}_{z} \hat{G}^{K}\left(\varepsilon^{\prime}-\varepsilon\right)\right. \\
& \left.+\hat{\tau}_{z} \hat{G}^{R}\left(\varepsilon^{\prime}\right) \hat{\tau}_{z} \hat{G}^{A}\left(\varepsilon^{\prime}-\varepsilon\right)+\hat{\tau}_{z} \hat{G}^{A}\left(\varepsilon^{\prime}\right) \hat{\tau}_{z} \hat{G}^{R}\left(\varepsilon^{\prime}-\varepsilon\right)\right]
\end{aligned}
$$

in which the trace $\operatorname{Tr}$ and the Pauli matrix $\hat{\tau}_{z}$ acts on the Nambu space [173].

To take into account the intrinsic damping of the oscillator, we additionally include a self-energy $\check{\Sigma}_{0}(\varepsilon)$ in Keldysh space. The self-energy is calculated by assuming that the environment is formed by a bath of independent harmonic oscillators (Caldeira-Leggett model) with a low-frequency linear dispersion for the spectral function, see for instance Refs. [83] and [174]. From this phenomenological model, one obtains the expressions [157]

$$
\begin{gathered}
\operatorname{Im} \Sigma_{0}^{R}(\varepsilon)=-\varepsilon / Q \\
\Sigma_{0}^{K}(\varepsilon)=-2 i \varepsilon \operatorname{coth}(\varepsilon) / Q,
\end{gathered}
$$

in which the coefficient $Q$ corresponds to the quality factor of the resonator.

Using the Dyson equation, we obtain the retarded, advanced, and Keldysh equations

$$
\begin{gathered}
D^{R, A}(\varepsilon)=\frac{2 \omega}{\varepsilon^{2}-\omega^{2}-2 \omega\left[\Pi^{R, A}(\varepsilon)+\Sigma_{0}^{R}(\varepsilon)\right]} \\
\simeq \frac{1}{\varepsilon-\omega \pm i \gamma_{\mathrm{tot}}}-\frac{1}{\varepsilon+\omega \pm i \gamma_{\mathrm{tot}}}, \\
D^{K}(\varepsilon)=D^{R}(\varepsilon)\left[\Pi^{K}(\varepsilon)+\Sigma_{0}^{K}(\varepsilon)\right] D^{A}(\varepsilon) \\
\simeq i \pi \frac{\Pi^{K}(\varepsilon)+\Sigma_{0}^{K}(\varepsilon)}{\gamma_{\mathrm{tot}}(\varepsilon)}[\delta(\varepsilon-\omega)+\delta(\varepsilon+\omega)],
\end{gathered}
$$

with the total damping rate given by

$$
\gamma_{\mathrm{tot}}(\omega)=-\operatorname{Im}\left[\Pi^{R}(\omega)+\Sigma_{0}^{R}(\omega)\right]=\gamma+\gamma_{0} .
$$

In Eq. (19) we have defined the intrinsic damping $\gamma_{0}=$ $-\operatorname{Im} \Sigma_{0}^{R}(\omega)=\omega / Q$ and the electromechanical damping $\gamma=$ $-\operatorname{Im} \Pi^{R}(\omega)$ due to the charge-vibration interaction. In Eq. (17) we expanded the retarded and advanced phonon Green's function close to $\varepsilon \simeq \omega$ taking into account the (small) frequency shift of the resonator $\Delta \omega=\operatorname{Re} \Pi^{R}(\omega)$.

\section{RESULTS FOR THE ELECTROMECHANICAL DAMPING}

We found that the electromechanical damping $\gamma$ in Eq. (19) due to the electron-vibration interaction can be divided according to the basic charge transport processes, i.e., reflections and transmissions, occurring in a N-QD-S system [171,172]. After some algebra, we cast the electromechanical damping as

$$
\gamma=\gamma_{\mathrm{NN}}+\gamma_{\mathrm{NS}}+\gamma_{\mathrm{SS}}
$$

in which the damping coefficients $\gamma_{\alpha \beta}$ with the labels $\alpha, \beta=$ $(\mathrm{N}, \mathrm{S})$ are associated to an inelastic process with an absorption or emission of one phonon with an incoming particle and an outgoing particle involving the leads $\alpha$ and $\beta$. Examples of such processes are given in Fig. 2.

The first term in Eq. (20) corresponds to reflections at the normal lead and can be divided into inelastic Andreev reflection (AR) and normal inelastic reflection (NR),

$$
\gamma_{\mathrm{NN}}=\gamma_{\mathrm{AR}}+\gamma_{\mathrm{NR}}
$$

A schematic view of inelastic AR and NR with an absorption of a phonon before an AR or NR is shown in Fig. 2(a) in which the excitation spectra of the normal and the superconducting lead around the Fermi momenta $k_{F}$ are shown as gray lines. Well below the superconducting gap, these processes are the only possible processes as an incoming electron can only be Andreev reflected or normal reflected.

The damping rate $\gamma_{\mathrm{AR}}$ and $\gamma_{\mathrm{NR}}$ can be written as the sum of elemental processes

$$
\begin{aligned}
& \gamma_{\mathrm{AR}}=\sum_{\nu s} s \gamma_{\nu \bar{v}}^{s}, \\
& \gamma_{\mathrm{NR}}=\sum_{\nu s} s \gamma_{\nu v}^{s},
\end{aligned}
$$

with the index $v= \pm=(e, h)$ for electrons and holes, respectively, and the notation $\bar{v}=-v$. The index $s= \pm$ refers to an inelastic process with an absorption $(s=+)$ and the emission $(s=-)$ of one phonon. As an example, $\gamma_{e h}^{s}$ corresponds to an inelastic AR with an absorption or emission of a phonon for an incoming electron and an outgoing hole at the normal lead. The damping rates $\gamma_{v v^{\prime}}^{s}$ in Eqs. (22) and (23) can be written as

$$
\gamma_{\nu \nu^{\prime}}^{s}(\omega)=\lambda^{2} \frac{\Gamma_{N}^{2}}{2} \int \frac{d \varepsilon}{2 \pi}\left|T_{\nu \nu^{\prime}}^{s}(\varepsilon)\right|^{2} f_{\nu}(\varepsilon)\left[1-f_{\nu^{\prime}}(\varepsilon+s \omega)\right],
$$

with the Fermi functions $f_{v}(\varepsilon)=1 /[1+\exp ((\varepsilon-v e V)) / T]$ for electron and holes, respectively, and the transmission functions

$$
\begin{aligned}
& T_{\nu \bar{v}}^{s}(\varepsilon)=G_{\nu}(\varepsilon) F^{*}(\varepsilon+s \omega)-F(\varepsilon) G_{\bar{\nu}}^{*}(\varepsilon+s \omega), \\
& T_{\nu \nu}^{s}(\varepsilon)=G_{\nu}(\varepsilon) G_{\nu}^{*}(\varepsilon+s \omega)-F(\varepsilon) F^{*}(\varepsilon+s \omega) .
\end{aligned}
$$

As discussed in Ref. [148] the transmission functions consist of a coherent sum of two amplitudes that are associated to the two possible paths in which the phonon is emitted or absorbed before or after a reflection. To the lowest order in the tunneling rates, the electromechanical dampings due to AR and NR are proportional to $\sim \Gamma_{S}^{2} \Gamma_{N}^{2}$ and $\sim \Gamma_{N}^{2}$, respectively. 

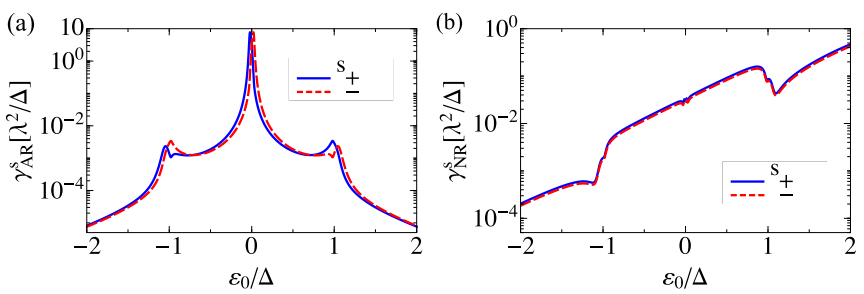

FIG. 4. Electromechanical damping associated to inelastic Andreev reflections $\gamma_{\mathrm{AR}}^{s}=\sum_{v} \gamma_{\mathrm{AR}}^{s v}$ and normal reflections $\gamma_{\mathrm{NR}}^{s}=$ $\sum_{v} \gamma_{\mathrm{NR}}^{s v}$ as a function $\varepsilon_{0}$ at $e V=3 \Delta$. The other parameters are $\Gamma_{N}=\Gamma_{S}=0.2 \omega$ and $\omega=0.05 \Delta$.

An example of the behavior of $\gamma_{\mathrm{AR}}$ and $\gamma_{\mathrm{NR}}$ is shown in Fig. 4. We analyzed in detail these processes in Ref. [148].

We now turn to discuss the term $\gamma_{\mathrm{NS}}$ in which a particle is exchanged between the normal contact (electron or hole) and the superconducting contact (quasiparticle). We can break up these terms in absorption or emission processes $(s)$ with an incoming electron or hole $(v)$

$$
\gamma_{\mathrm{NS}}=\sum_{s v} s \gamma_{\mathrm{NS}}^{s v}
$$

with

$$
\begin{aligned}
\gamma_{\mathrm{NS}}^{s v}= & 16 \lambda^{2} \Gamma_{N} \Gamma_{S} \int \frac{d \varepsilon}{2 \pi} \mathcal{T}_{v}^{s}(\varepsilon) \rho_{S}(\varepsilon+s \omega) \\
& \times f_{v}(\varepsilon)\left[1-f_{0}(\varepsilon+s \omega)\right] .
\end{aligned}
$$

In Eq. (28) we have introduced the superconducting density of states defined as $\rho_{S}(\varepsilon)=\theta\left(\varepsilon^{2}-\Delta^{2}\right) \varepsilon / \sqrt{\varepsilon^{2}-\Delta^{2}}$ normalized to the one in the normal phase, and the transmission function

$$
\begin{aligned}
\mathcal{T}_{\nu}^{s}(\varepsilon)= & \left|T_{\nu \nu}^{s}(\varepsilon)\right|^{2}+\left|T_{\nu \bar{v}}^{s}(\varepsilon)\right|^{2}+\frac{2 \Delta}{\varepsilon+s \omega} \\
& \times \operatorname{Re}\left[T_{\nu v}^{s}(\varepsilon) T_{\nu \bar{\nu}}^{s}(\varepsilon)^{*}\right] .
\end{aligned}
$$

The processes described by Eq. (28) admit two equivalent descriptions: For instance the term for $v=e$ can be seen as an impinging electron from the normal lead $N$ to the superconductor $S$, leaving a hole in the lead $N$, or it can be seen as an impinging quasiparticle from the superconductor $S$ transmitted as an hole in the normal lead $N$.

Comparing the results Eqs. (28) and (29) with the ones reported in literature [171,172], it turns out that the term in Eq. (29) proportional to $\left|T_{v v}(\varepsilon)\right|^{2}$ corresponds to the inelastic direct tunneling of quasiparticles (DT) and the term with $\left|T_{\nu \bar{v}}(\varepsilon)\right|^{2}$ corresponds to branch crossing (BC), according to the terminology introduced in the BTK model [172]. When an electron is transmitted via inelastic DT, it occupies an electronlike branch on the superconductor. Branch crossing refers to the transmission of the electron on the holelike branch of the superconductor. Examples of processes corresponding to inelastic DT and BC for an incoming electron from the normal metal are sketched in Fig. 2(b), in which a phonon is absorbed when the electron is transmitted to the superconductor. To the leading order in the tunneling rates, DT and BC are proportional to $\Gamma_{S} \Gamma_{N}$ and $\Gamma_{S}^{2} \Gamma_{N}^{2}$, respectively. The last term in Eq. (29) is proportional to the product of two transmission functions and can be interpreted as tunneling via an intermediate state [171] and scales as $\Gamma_{N} \Gamma_{S}^{2}$.

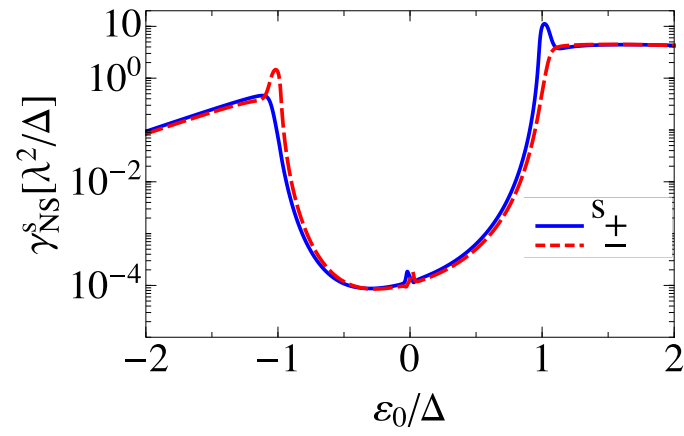

FIG. 5. Electromechanical damping associated to the inelastic tunneling between $N$ and $S \gamma_{\mathrm{NS}}^{s}=\sum_{v} \gamma_{\mathrm{NS}}^{s v}$ for one impinging electron $(v=+=e)$ as a function $\varepsilon_{0}$ at $e V=3 \Delta$. The parameters are $\Gamma_{N}=$ $\Gamma_{S}=0.2 \omega$ and $\omega=0.05 \Delta$.

An example of the behavior of $\gamma_{\mathrm{NS}}^{s}=\sum_{v} \gamma_{\mathrm{NS}}^{s v}$ is shown in Fig. 5. By comparison of Fig. 5 with Fig. 4 we notice that the damping associated to the inelastic tunneling of quasiparticles is negligible when the dot's level is well inside the gap $\left|\varepsilon_{0}\right| \ll$ $\Delta$ and becomes relevant when $\left|\varepsilon_{0}\right| \sim \Delta$ whereas the damping associated to inelastic Andreev reflections dominates at low energy $\left|\varepsilon_{0}\right| \ll \Delta$. We will explain this behavior in the next section.

Finally, for completeness, we report the expression for the reflections involving the quasiparticles of the superconductor. This term is given by

$$
\gamma_{\mathrm{SS}}=\sum_{\nu s} s \gamma_{\mathrm{SS}}^{s v}
$$

with each term referring to the individual process of emission or absorption for electron/hole. They read

$$
\begin{aligned}
\gamma_{S S}^{s v}= & 8 \lambda^{2} \Gamma_{S}^{2} \int \frac{d \varepsilon}{2 \pi} \mathcal{R}_{v}^{s}(\varepsilon) \rho_{S}(\varepsilon) \rho_{S}(\varepsilon+s \omega) \\
& \times f_{0}(\varepsilon)\left[1-f_{0}(\varepsilon+s \omega)\right]
\end{aligned}
$$

and the reflection function

$$
\begin{aligned}
\mathcal{R}_{\nu}^{s}(\varepsilon)= & \mathcal{T}_{\nu}^{s}(\varepsilon)+\operatorname{Re}\left[\frac{\Delta}{\varepsilon} T_{\nu v}(\varepsilon) T_{\bar{\nu} \nu}^{*}(\varepsilon)+\frac{\Delta^{2}}{\varepsilon(\varepsilon+s \omega)}\right. \\
& \left.\times\left(T_{\nu v}(\varepsilon) T_{\bar{\nu} \bar{v}}^{*}(\varepsilon)+T_{\nu \bar{v}}(\varepsilon) T_{\bar{\nu} \nu}^{*}(\varepsilon)\right)\right] .
\end{aligned}
$$

The damping contribution associated to the quasiparticle reflections in the superconductor $\gamma_{\mathrm{SS}}$ is independent of the applied voltage. Moreover, for low temperatures compared to the superconducting gap $T \ll \Delta$, the population of quasiparticles above the gap is exponentially small such that we can disregard $\gamma_{\text {SS }}$ for the rest of our discussion.

\section{RESULTS FOR THE PHONON OCCUPATION}

Applying a bias voltage, the electron current drives the oscillator to a nonequilibrium state with phonon occupation

$$
n=\left\langle b^{\dagger} b\right\rangle=-\frac{1}{2}+\frac{i}{8 \pi} \int d \varepsilon D^{K}(\varepsilon) \simeq-\frac{\operatorname{Im} \Pi^{K}(\omega)}{4 \gamma(\omega)}-\frac{1}{2} .
$$


In the limit $\gamma_{\text {tot }} \ll\left(\omega, \Gamma_{N}, \Gamma_{S}, T, e V\right)$ and separating the contribution of the intrinsic damping and the damping due to the charge-vibration interaction, the phonon occupation can be written as

$$
\bar{n}=\frac{\gamma_{\mathrm{AR}} n_{\mathrm{AR}}+\gamma_{\mathrm{NS}} n_{\mathrm{NS}}+\left(\gamma_{\mathrm{NR}}+\gamma_{\mathrm{SS}}+\gamma_{0}\right) n_{B}(\omega)}{\gamma_{\mathrm{AR}}+\gamma_{\mathrm{NS}}+\gamma_{\mathrm{NR}}+\gamma_{\mathrm{SS}}+\gamma_{0}}
$$

with the Bose function $n_{B}(\omega)=[\exp (\omega / T)-1]^{-1}$. The nonequilibrium occupation due to inelastic ARs reads [148]

$$
n_{\mathrm{AR}}=\frac{1}{\gamma_{\mathrm{AR}}} \sum_{\nu s} s \gamma_{\nu \bar{v}}^{s} n_{B}(\omega+\nu s 2 e V),
$$

whereas the nonequilibrium occupation $n_{\mathrm{NS}}$ is given by

$$
n_{\mathrm{NS}}=\frac{1}{\gamma_{\mathrm{NS}}} \sum_{\nu s} s \gamma_{\mathrm{NS}}^{s v} n_{B}(\omega+\nu s e V)
$$

with the Bose function shifted by $e V$ due to the tunneling of quasiparticles. Notice that the normal reflection (NR) and the quasiparticle reflections (SS) can drive the oscillator only to the thermal equilibrium.

For low temperature compared to the gap $T \ll \Delta$ and for the case $\gamma_{0} \ll \gamma_{\mathrm{NR}}$, we can approximate $\gamma_{\mathrm{NR}}+\gamma_{\mathrm{SS}}+\gamma_{0} \simeq$ $\gamma_{\mathrm{NR}}$. Moreover, in the high voltage limit $e V \gg T$ and for positive bias $e V>0$, we have essentially that electrons from the normal lead are moving to the right superconducting lead (or equivalently the holes move from the right to the left). In this case, we can approximate the expression for $\bar{n}$ as

$$
\bar{n} \simeq \frac{\gamma_{e h}^{-}+\gamma_{\mathrm{NS}}^{-e}+\gamma_{\mathrm{NR}} n_{B}(\omega)}{\left(\gamma_{e h}^{+}+\gamma_{e h}^{-}\right)+\left(\gamma_{\mathrm{NS}}^{+e}-\gamma_{\mathrm{NS}}^{-e}\right)+\gamma_{\mathrm{NR}}} .
$$

Equation (37) generalizes the main result in Ref. [148] as it takes into account the effects of the quasiparticles on the nonequilibrium phonon occupation of the mechanical oscillator.

\section{A. Single mode phonon occupation}

Figure 6(a) shows an example of the phonon occupation $\bar{n}$ as a function of $\varepsilon_{0}$ and source-drain voltage $e V$ for a single mode with frequency $\omega \ll \Delta$ and a temperature $T=10 \omega$. As anticipated in the introduction, we have: (i) regions of ground state cooling $\bar{n} \ll 1$, (ii) regions of strong energy pumping into the resonator $\bar{n} \gg 1$, and (iii) regions of a mechanical instability in which the total electromechanical damping becomes negative $\gamma+\gamma_{0}<0$ and our perturbative method breaks down. In analogy to previous results in Ref. [66], one expects that, going beyond the perturbative approach for the charge-vibration interaction and taking into account the anharmonicity of the resonator, such regions can host self-sustained oscillations. In Fig. 6(b) we show at fixed bias voltage the total damping and the contributions of $\gamma_{\mathrm{AR}}$ and $\gamma_{\mathrm{NS}}$ to the electromechanical damping that are responsible for the instability, i.e., $\gamma_{\mathrm{AR}}<0$ or $\gamma_{\mathrm{NS}}<0$.

The first striking observation in Fig. 6(a) is the weakly dependence of the phonon occupation $\bar{n}$ on the bias voltage whereas $\bar{n}$ is strongly dependent on $\varepsilon_{0}$. In other words, the phonon occupation is determined by the bare dot's level $\varepsilon_{0}$, or the gate voltage, whereas no additional features appear at $e V \sim \Delta$. This behavior can be explained as we assumed a sharp resonant regime with a small coupling to the normal lead
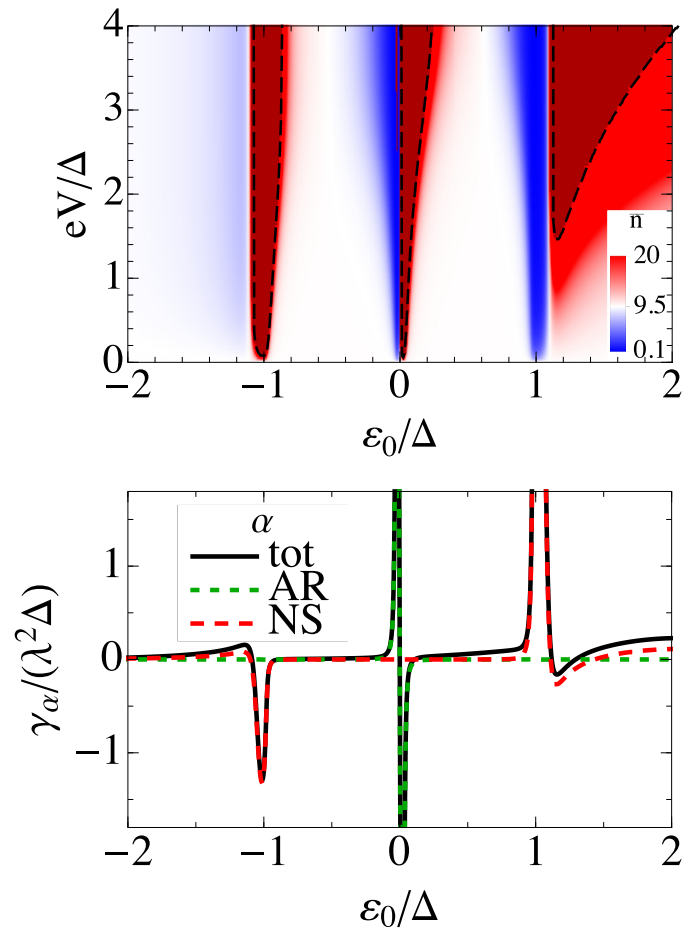

FIG. 6. (a) Phonon occupation as a function of bias voltage $e V$ and gate voltage $\varepsilon_{0}$ at $T=10 \omega$. The parameters are $\Gamma_{N}=\Gamma_{S}=$ $0.2 \omega, \lambda=0.1 \omega, \gamma_{0}=10^{-6} \omega$, and $\omega=0.05 \Delta$. The dark red regions surrounded by dashed lines indicate a mechanical instability with $\gamma_{\text {tot }}<0$. (b) Mechanical damping $\gamma_{\text {tot }}, \gamma_{\mathrm{AR}}$, and $\gamma_{\mathrm{NS}}$ as a function of gate voltage at $e V=2 \Delta$.

$\Gamma_{N}=0.1 \omega \ll \Delta$. As discussed in Sec. II A, the Andreev levels $E_{A}$ are formed within the superconducting gap whose position depends on $\varepsilon_{0}$ (and the coupling to the superconductor $\Gamma_{S}$ ) and whose small broadening is set by $\Gamma_{N}$. This translates into sharp peaks entering in the transmission functions in Eqs. (25), (26), and (29) and yield a saturation for the damping rates $\gamma_{\mathrm{AR}}, \gamma_{\mathrm{NR}}$, and $\gamma_{\mathrm{NS}}$ as increasing the bias voltage. Thus we divide the following discussion about the phonon occupation of Fig. 6(a) into the deep subgap regime with $\left|\varepsilon_{0}\right| \ll \Delta$ and a regime close to the gap $\left|\varepsilon_{0}\right| \sim \Delta$. This scale separation is valid for $\omega \ll \Delta$.

In the first region at low energy, $\left|\varepsilon_{0}\right| \ll \Delta$, inelastic AR dominates (see Fig. 4). This subgap regime was discussed in Ref. [148] in which we showed that resonances in both amplitudes of the transmission function in Eq. (25) as well as interference between the two amplitudes result in: (i) an enhancement of the absorption rates compared to the emission rates for $\varepsilon_{0}<0$ leading to ground-state cooling, (ii) a large phonon occupation eventually approaching a mechanical instability for $\varepsilon_{0}>0$. Here, we extend the previous result showing that $\bar{n}$ is controlled by the gate voltage even for bias voltages larger than the superconducting gap $e V>\Delta$.

By contrast, the inelastic tunneling of quasiparticles strongly controls the phonon occupation when the gate voltage approaches the superconducting gap $\left|\varepsilon_{0}\right| \simeq \Delta$ (see Fig. 6). Indeed, in this region, inelastic quasiparticle tunnelings are the leading processes as shown in Fig. 5. These processes, similarly to the inelastic ARs, yield a similar behavior of the nonequilibrium occupation with alternating regions of ground-state cooling, energy "heating", and instability. The 
line separating the ground state cooling $\bar{n} \ll 1$ from the region $\bar{n} \gg 1$ is a narrow range in which the resonator is close to the thermal equilibrium. These results can be explained by considering the subtle interplay between the density of states $\rho_{d}(\varepsilon)$ of the quantum dot [see Fig. 3(a)], that acts as energy filter via the transmission coefficient $\mathcal{T}_{v}^{s}(\varepsilon)$ in Eq. (26), and the behavior of the superconducting density of states with an energy gap and a divergence at $\varepsilon=\Delta$. The basic idea is schematically reported in Fig. 7. Hereafter we discuss in detail the positive part $\varepsilon_{0}>0$ at $\varepsilon_{0} \simeq \Delta$. Similar arguments apply for the negative part $\varepsilon_{0}<0$ at $\varepsilon_{0} \simeq-\Delta$.

We start the discussion with the case shown in Fig. 7(a) when the Andreev states are well formed inside the dot and the energy level $\varepsilon_{0}<\Delta$. Tunneling from $N$ to $S$ with phonon absorption or phonon emission are possible for electrons with the appropriate energy threshold, namely $\varepsilon \geqslant \Delta-\omega$ or $\varepsilon \geqslant$ $\Delta+\omega$, respectively. However, the density of states $\rho_{d}(\varepsilon)$ [and hence the transmission function $\left.\mathcal{T}_{v}^{s}(\varepsilon)\right]$ is sharply peaked at the Andreev levels such that one can assume that electron are essentially injected at energy $\varepsilon=\left|E_{A}\right|$. In this case shown in Fig. 7(a), the emission of a phonon is simply blocked due the superconducting gap.

Increasing $\varepsilon_{0}$, we approach the region $\varepsilon_{0}>\Delta$. Here, beyond Andreev levels inside the gap, a broadened peak appears in $\rho_{d}(\varepsilon)$ - and in the transmission $\mathcal{T}_{v}^{s}(\varepsilon)$-above the gap, reminiscent of the bare dot's level [see Figs. 3(a) and 7(b)]. In this case, beyond the fact that phonon emission is still blocked due the superconducting gap, the absorption rate is in addition enhanced due to the increase in $\rho_{d}(\varepsilon)$ and $\mathcal{T}_{v}^{s}(\varepsilon)$ at $\varepsilon>\Delta$.

Finally, further increasing $\varepsilon_{0}$, the peak of the functions $\rho_{S}(\varepsilon)$ or $\mathcal{T}_{v}^{s}(\varepsilon)$ moves far away from the gap edge such that the condition $\varepsilon_{0}=\Delta+\omega$ is reached. In this case, electrons from the normal lead $N$ are again filtered by the dot at energy $\varepsilon=\varepsilon_{0}$ and phonon emission are greatly enhanced in this case due to the divergence of $\rho_{S}(\varepsilon)$ at the gap edge, viz. $\gamma_{\mathrm{NS}}^{-}>\gamma_{\mathrm{NS}}^{+}$, leading to the instability.

\section{B. Multimode phonon ground-state cooling}

In this section, we illustrate that several nondegenerate mechanical modes can be cooled to the ground state in the
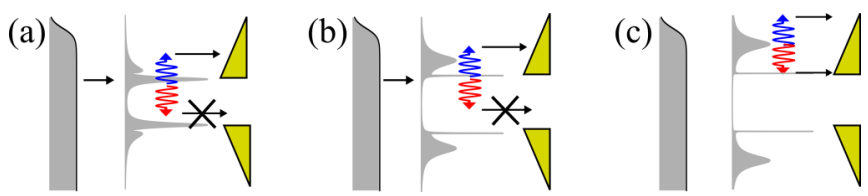

FIG. 7. Schematic view of absorption and emission processes due to inelastic quasiparticle tunneling for the case $|e V-\Delta| \gg T$. In (a) and (b) the emission of a vibrational energy quantum is essentially suppressed by the superconducting gap. Figure (a) represents the case of $\varepsilon_{0}<\Delta$ and $E_{A}<\Delta$. Figure (b) represents the case of $\varepsilon_{0}>\Delta$ but still $E_{A}<\Delta$, in which the density of states above the gap is enhanced due to the resonant level of the dot leading to a further increase of the absorption processes. In (c), for $\varepsilon_{0}+\omega \geqslant \Delta$, no Andreev bound states are formed in the dot and the emission is enhanced due to the singular behavior of the superconducting density of state at the energy $\varepsilon=\Delta$.
N-QS-S system. We generalized the Hamiltonian Eq. (1) considering several vibrational eigenmodes of the resonator. The frequencies of these vibrational modes are denoted by $\omega_{m}$ and the bosonic annihilation and creation operators for each mode by $\hat{b}_{m}$ and $\hat{b}_{m}^{\dagger}$. The Hamiltonian reads:

$$
\begin{aligned}
\hat{H}= & \hat{H}_{N}+\hat{H}_{S}+\hat{H}_{t}+\varepsilon_{0} \hat{n}_{d} \\
& +\sum_{m}\left[\lambda_{m} \hat{n}_{d}\left(\hat{b}_{m}^{\dagger}+\hat{b}_{m}\right)+\omega_{m} \hat{b}_{m}^{\dagger} \hat{b}_{m}\right] .
\end{aligned}
$$

To illustrate the multimode cooling, we assume a linear low-frequency spectrum by $\omega_{m}=m \omega$ with $m=1,2, \ldots, N$ although this assumption is not essential. The calculation of the multimode phonon occupation can be limited by considering a finite number of modes, as we have a natural cutoff given by the temperature. For the case $T=10 \omega$ we choose $N=7$ modes. High-frequency modes with $\omega \gtrsim T$ are close to the ground state. The nonequilibrium value $\bar{n}_{k}$ for each mode is calculated by Eq. (34) from which we can plot the total mechanical energy defined by $E_{\text {tot }}=\sum_{m=1}^{N} \omega_{m} \bar{n}_{m}$. Such a approach holds since, in the regime of weak charge-vibration coupling, we can neglect the intercorrelation effect between the different modes.

In Fig. 8 we show the multimode phonon occupation at bias voltage larger than the gap $e V>\Delta$ and as a function of $\varepsilon_{0}$. Similarly to the discussion in Sec. IV A of the phonon occupation for a single mode, we found that the total energy $E_{\text {tot }}$ has a weak bias-voltage dependence and its behavior is controlled by the gate voltage $\varepsilon_{0}$.

As can be seen from Fig. 8, the thermal equilibrium value of the total mechanical energy $E_{\mathrm{tot}} \approx 57.2 \omega$ is approached if the gate voltage is much larger than the gap, $\left|\varepsilon_{0}\right| \gg \Delta$. Figure 8 has a similar behavior as the single mode results $\bar{n}$ with features in the deep subgap region $\left|\varepsilon_{0}\right| \ll \Delta$ and close to the gap edges $\left|\varepsilon_{0}\right| \sim \Delta$. Around these ranges, the total energy shows dips below the thermal equilibrium value followed by sharp increases of the multimode phonon occupation.

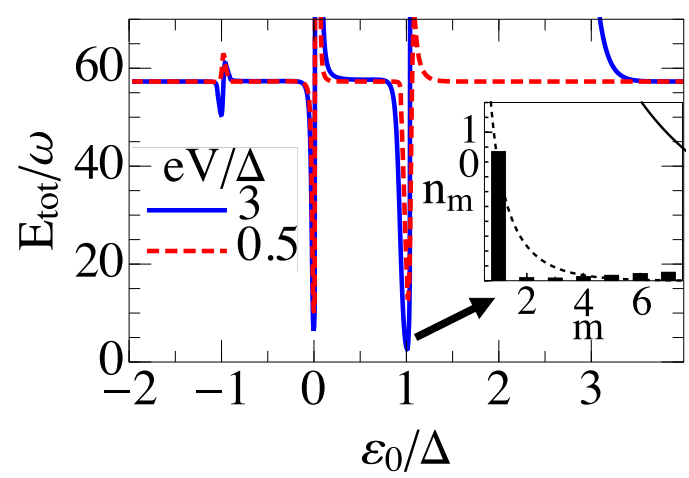

FIG. 8. Multimode phonon occupation. Total mechanical energy $E_{\mathrm{tot}}=\sum_{m} \hbar \omega_{m} \bar{n}_{m}$ for $m=7$ modes as a function gate voltage $\varepsilon_{0}$ at $T=10 \omega$ for two different bias voltages. The parameters are $\Gamma_{N}=$ $\Gamma_{S}=0.2 \omega, \omega=0.01 \Delta, \gamma_{0}=10^{-6} \omega$. The total mechanical energy at equilibrium is given by $E_{\mathrm{tot}} \approx 57.2 \omega$. Inset: Distribution $n_{m}$ for each mode at the minimum of the total energy. The solid line is $n_{B}(\omega)$ at $T=10 \omega$. The dashed line corresponds to the Bose distribution $n_{B}\left(\omega_{m}\right)$ at the effective temperature $T=1.2 \omega$. 
When the gate voltage is close to $\varepsilon_{0} \simeq-\Delta$ and for $e V=$ $3 \Delta$, the total energy has a small dip (weak cooling) followed by an increase due to inelastic tunneling of quasiparticles. In this case, since the temperature is small compared to the superconductor gap $T \ll \Delta$, the states in the superconductor are almost filled and the phonon occupations of the several modes are only slightly reduced.

As discussed in a previous work in Ref. [148], for negative $\varepsilon_{0}<0$ and $\left|\varepsilon_{0}\right| \ll \Delta$, all the vibrational modes are strongly cooled by inelastic-vibration assisted Andreev reflections. Due to an interference mechanism, such a cooling is possible without matching a resonant condition between levels of the quantum dot and the vibrational frequency. Here we demonstrate that this mechanism is working even at bias voltage larger than the gap.

Interestingly, the phonon occupation of several modes is strongly reduced even closely below the superconducting gap, for $\varepsilon_{0} \simeq \Delta$, where the dominant processes are the inelastic tunneling of quasiparticles. The explanation is similar to the discussion of the previous section for the damping (see Fig. 7).

\section{RESULTS FOR THE CURRENT}

In this section, we calculate the current to the leading order in the charge-vibration coupling for a single mode with frequency $\omega$. Using the spinor notation in Nambu space in the normal lead $\Psi_{k}^{\dagger}=\left(c_{k \uparrow}^{\dagger} c_{-k \downarrow}\right)$ and the number operator $\hat{N}=\frac{1}{2} \sum_{k} \Psi_{k}^{\dagger} \hat{\tau}_{z} \Psi_{k}$, with the Pauli matrix $\hat{\tau}_{z}$, see Ref. [173], the current through the normal lead $I=-e\langle d \hat{N} / d t\rangle$ can be expressed as

$$
I=\frac{e}{2 \hbar} \int \frac{d \varepsilon}{2 \pi} \operatorname{Re} \operatorname{Tr}\left[\hat{\tau}_{z}\left(\hat{\mathcal{G}}^{K}(\varepsilon) \hat{\Sigma}_{N}^{A}+\hat{\mathcal{G}}^{R}(\varepsilon) \hat{\Sigma}_{N}^{K}(\varepsilon)\right)\right]
$$

in which $\hat{\mathcal{G}}^{K}(\varepsilon)$ and $\hat{\mathcal{G}}^{R}(\varepsilon)$ refer to the dot Green's functions and they are defined similarly as in Sec. II A. They correspond to the exact Green's function including the coupling to the leads and the charge-vibration interaction. To calculate the current in Eq. (39) we perform a perturbation expansion of these Green's functions to the leading order in the charge-vibration coupling $\lambda[115,157]$. Hence the current can be decomposed into an elastic current, an elastic correction, and an inelastic term as

$$
I=I_{0}+I_{\mathrm{ec}}^{\left(\lambda^{2}\right)}+I_{\mathrm{in}}^{\left(\lambda^{2}\right)}
$$

with

$$
\begin{gathered}
I_{0}=e \int \frac{d \varepsilon}{2 \pi} \operatorname{Re} \operatorname{Tr}\left[\hat{\tau}_{z}\left(\hat{G}^{K} \hat{\Sigma}_{N}^{A}+\hat{G}^{R} \hat{\Sigma}_{N}^{K}\right)\right] \\
I_{\mathrm{ec}}^{\left(\lambda^{2}\right)}= \\
e \int \frac{d \varepsilon}{2 \pi} \operatorname{Re} \operatorname{Tr}\left[\hat { \tau } _ { z } \left(\hat{G}^{R} \hat{\Sigma}_{\mathrm{vib}}^{R} \hat{G}^{K} \Sigma_{N}^{A}+\hat{G}^{K} \hat{\Sigma}_{\mathrm{vib}}^{A} \hat{G}^{A} \hat{\Sigma}_{N}^{A}\right.\right. \\
\left.\left.+\hat{G}^{R} \hat{\Sigma}_{\mathrm{vib}}^{R} \hat{G}^{R}\left(\hat{\Sigma}_{N}^{R}-\hat{\Sigma}_{N}^{A}+\hat{\Sigma}_{S}^{R}-\hat{\Sigma}_{S}^{A}\right) \hat{G}^{A} \hat{\Sigma}_{N}^{K}\right)\right], \\
I_{\mathrm{in}}^{\left(\lambda^{2}\right)}=e \int \frac{d \varepsilon}{2 \pi} \operatorname{Re} \operatorname{Tr}\left[\hat{\tau}_{z}\left(\hat{G}^{R} \hat{\Sigma}_{\mathrm{vib}}^{K} \hat{G}^{A} \hat{\Sigma}_{N}^{A}+\hat{G}^{R} \hat{\Sigma}_{\mathrm{vib}}^{R} \hat{G}^{A} \hat{\Sigma}_{N}^{K}\right)\right],
\end{gathered}
$$

and the self-energies associated to the charge-vibration interaction $\left(\sim \lambda^{2}\right)$

$$
\begin{gathered}
\hat{\Sigma}_{\mathrm{vib}}^{R / A}(\varepsilon)=i \frac{\lambda^{2}}{2} \int \frac{d \varepsilon^{\prime}}{2 \pi} D^{R / A}\left(\varepsilon^{\prime}\right) \hat{\tau}_{z} \hat{G}^{K}\left(\varepsilon-\varepsilon^{\prime}\right) \hat{\tau}_{z} \\
-\hat{\tau}_{z} \operatorname{Tr}\left[\hat{\tau}_{z} \hat{G}^{K}\left(\varepsilon^{\prime}\right)\right] D^{R, A}(0), \\
\hat{\Sigma}_{\mathrm{vib}}^{K}(\varepsilon)=i \frac{\lambda^{2}}{2} \int \frac{d \varepsilon^{\prime}}{2 \pi} \sum_{\xi=R, A, K} D^{\xi}\left(\varepsilon^{\prime}\right) \hat{\tau}_{z} \hat{G}^{\xi}\left(\varepsilon-\varepsilon^{\prime}\right) \hat{\tau}_{z} .
\end{gathered}
$$

The elastic current $I_{0}$ shows a peak at $\varepsilon_{0}=0$ as a function of the gate voltage which is associated to the Andreev reflections. In the deep subgap regime, this current can be simplified to

$$
I_{0}=8 e \Gamma_{N}^{2} \int d \varepsilon|F(\varepsilon)|^{2}\left[f_{+}(\varepsilon)-f_{-}(\varepsilon)\right] .
$$

In addition, $I_{0}$ increases close to the gap edges at $\varepsilon_{0} \simeq|\Delta|$ of the gate voltage, where quasiparticle tunneling occurs and contributes to transport.

The term $I_{\mathrm{ec}}^{\left(\lambda^{2}\right)}$ represents the corrections to the elastic part and shows no additional features with respect to $I_{0}$. On the other hand, the inelastic term of the current $I_{\text {in }}^{\left(\lambda^{2}\right)}$ has different features. This term can be approximated in the high-voltage limit as

$$
I_{\mathrm{in}}^{\left(\lambda^{2}\right)} / e \simeq(\langle\hat{n}\rangle+1)\left(\gamma_{\mathrm{AR}}^{-}+\gamma_{\mathrm{NS}}^{-}\right)+\langle\hat{n}\rangle\left(\gamma_{\mathrm{AR}}^{+}+\gamma_{\mathrm{NS}}^{+}\right)
$$

for $e V \gg T$. The inelastic current is directly related to the average phonon occupation $\langle\hat{n}\rangle$. In the following, we discuss two different cases. In the following, we discuss two different limits. In the first case, we consider the vibrational states at thermal equilibrium $\langle\hat{n}\rangle=n_{B}(\omega)$ which corresponds to the externally damped harmonic oscillator. In the second case we assume that the vibration is driven in a strong nonequilibrium state $\bar{n}$ given by Eq. (34) with small intrinsic damping $\gamma_{0} / \omega \ll 1$. This second case corresponds to the undamped harmonic oscillator and the phonon occupation is practically controlled by the charge passing through the dot (viz. the bias voltage). Notice however that, for vanishing intrinsic damping $\gamma_{0}=0$, $\bar{n}$ is of order $\lambda^{0} \sim 1$ such that the inelastic corrections to the current are still of the order $\lambda^{2}$. More redefined numerical treatments as the self-consistent approach based by solving numerically the coupled Dyson equations for the electron and phonon's Green's functions is beyond the aim of this work [175-179]. As an example of this comparison, we show the current as a function of $\varepsilon_{0}$ at large bias voltage $e V=10 \Delta$ for a resonator at thermal equilibrium $\langle\hat{n}\rangle=n_{B}(\omega)$ in Fig. 9(a) and in a nonequilibrium state $\langle\hat{n}\rangle=\bar{n}$ in Fig. 9(b).

In both cases, we observe that the inelastic current shows distinct features. At small gate bias $\left(\varepsilon_{0} \rightarrow 0\right)$, the peaks at $\varepsilon_{0}=-\omega / 2$ and $\varepsilon_{0}=\omega / 2$ are associated to the absorption and emission, respectively, of a phonon during inelastic, vibration assisted Andreev reflections [148]. Additionally to these peaks, we also found peaks close to the gap edges where inelastic quasiparticles tunneling give a contribution to transport at $E_{A}= \pm|\Delta \pm \omega|$, which can be explained by the same argument discussed at the end of Sec. IV A (see Fig. 7). The peaks at $E_{A}=|\Delta-\omega|$ correspond to the absorption of one phonon and $E_{A}=-|\Delta-\omega|$ to the emission of one phonon. We notice that the role of the peaks at the negative part 

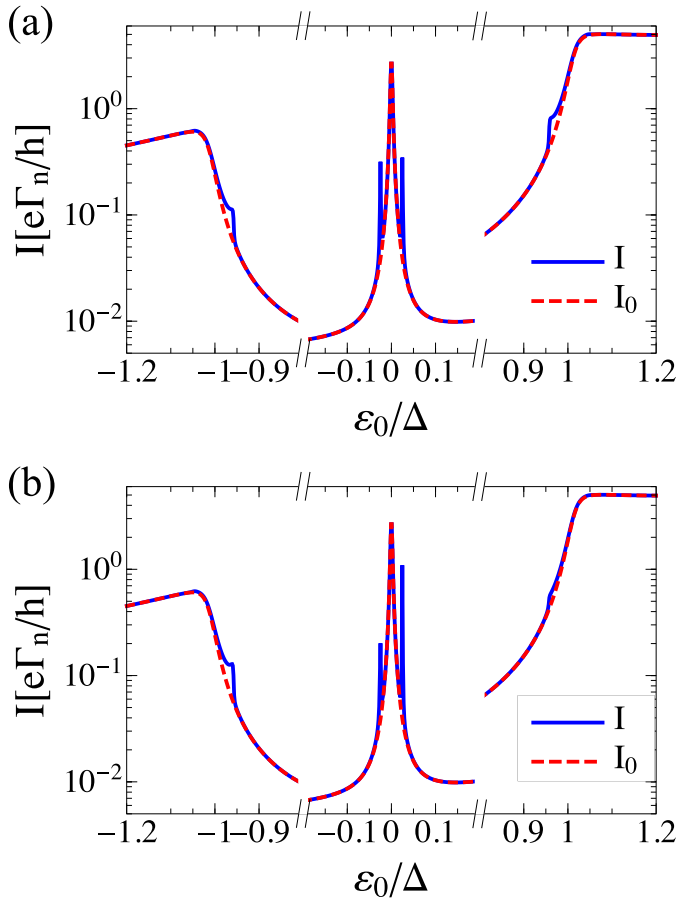

FIG. 9. Total current $I$ and elastic current $I_{0}$ as a function of $\varepsilon_{0}$ at $\omega=0.05 \Delta$. In (a), the resonator is in thermal equilibrium with large intrinsic damping such that $\bar{n}=n_{B}(\omega)$. In (b), the resonator is in a nonequilibrium state with $\lambda=0.015 \omega$ and $\gamma_{0}=10^{-4} \omega$. The other parameters are $\Gamma_{N}=0.015 \omega, \Gamma_{S}=0.05 \omega, T=10 \omega$, and $e V=10 \Delta$.

$\left(\varepsilon_{0}<0\right)$ and positive part $\left(\varepsilon_{0}>0\right)$ is inverted in comparison to peaks associated to the ARs.

The difference behavior of the peaks in Figs. 9(a) and 9(b) is due to the two different regimes for the phonon occupation. When the vibration is at thermal equilibrium as in Fig. 9(a), both kinds of the peaks associated to the inelastic processes involving ARs and quasi-particles are almost of the same height, albeit in the limit $n_{B}(\omega)+1 \simeq n_{B}(\omega)$. By contrast, in Fig. 9(b) the resonator is in a nonequilibrium state and the paired sidepeaks are of different height: The peaks which are associated to the phonon absorption are suppressed compared the thermal case whereas the peaks associated to the phonon emission are enhanced. In other words, the asymmetry of the peaks reflects the behavior of the phonon occupation shown in Fig. 6. This result implies that the heights of the inelastic peaks lying in two different ranges of the gate voltage are correlated and they can be readily used to detect the nonequilibrium state of the resonator. Then, using the same argument discussed in Ref. [148], the ratio between the brightness of paired peaks-defined as the underlying area for each peak-can be used to extract information about the phonon occupation of the nonequilibrated resonator.

\section{CONCLUSION}

We studied the phonon occupation and the quantum transport of a quantum dot embedded between a normal-conducting and a superconducting lead showing that the vibrational states of the resonator are controlled by the current. In comparison to our previous work in Ref. [148], we consider a finite superconducting gap and showed that quasiparticle tunneling close to the superconducting gap strongly affects the phonon occupation. When the energy of the resonant level is well inside the superconducting gap, inelastic vibration-assisted Andreev reflections drive the resonator to a nonequilibrium state. At gate voltages close to the superconducting gap edge, inelastic tunneling of quasiparticles control the state of the resonator. As a result we obtained that both inelastic vibration-assisted Andreev reflections and inelastic tunneling of quasiparticles can cool the vibrational state of the resonator to the ground state even for several vibrational modes.

Moreover, we found that quasiparticles inelastic tunneling yields peaks even inside the subgap transport region, close to the gap and far from the peaks associated to the Andreev inelastic reflections. According to our theory, the two kind of peaks are correlated and this property can be readily exploited for the spectroscopy of the vibrational modes. Similar inelastic processes were observed in recent experiments [144-147] suggesting that our proposal is within the reach of current research.

The system allows in principle to prepare vibrational modes in the quantum regime by cooling via electron transport. Ground state cooling of the mechanical motion can be useful in view of the application of such devices as quantum, ultrasensitive detectors of mass, charge, spin and force at the nanoscales, or for fundamental studies of quantum mechanics, viz. the observation of quantum effects in large mechanical systems formed by millions of atoms. Our method can be used to initialize the quantum state of the mechanical vibrations of a carbon nanomechanical resonator and subsequently to address the relaxation dynamics and the dynamical crossover from the quantum regime towards the classical regime. Further possible applications concern the cooling of spatially separated nanomechanical resonators and other nonlocal heat transport phenomena.

\section{ACKNOWLEDGMENTS}

We acknowledge J. C. Cuevas, E. Scheer, S. Andergassen, and T. Pietsch for interesting discussions and useful comments. This research was supported by the Zukunftskolleg of the University of Konstanz and by the DFG through the collaborative research center SFB 767.
[1] Y. V. Nazarov and Y. M. Blanter, Quantum Transport: Introduction to Nanoscience, 1st ed. (Cambridge University Press, New York, 2009).

[2] J. C. Cuevas and E. Scheer, Molecular Electronics: An introduction to Theory and Experiment, 1st ed. (World Scientific, Singapore, 2010).
[3] M. Galperin, M. A. Ratner, and A. Nitzan, J. Phys.: Condens. Matter 19, 103201 (2007).

[4] S. V. Aradhya and L. Venkataraman, Nat. Nanotechnol. 8, 399 (2013).

[5] H. Park, J. Park, A. K. L. Lim, E. H. Andersen, A. P. Alivisatos, and P. L. McEuen, Nature (London) 407, 57 (2000). 
[6] L. H. Yu, Z. K. Keane, J. W. Ciszek, L. Cheng, M. P. Stewart, J. M. Tour, and D. Natelson, Phys. Rev. Lett. 93, 266802 (2004).

[7] X. H. Qiu, G. V. Nazin, and W. Ho, Phys. Rev. Lett. 92, 206102 (2004).

[8] S. W. Wu, G. V. Nazin, X. Chen, X. H. Qiu, and W. Ho, Phys. Rev. Lett. 93, 236802 (2004).

[9] A. N. Pasupathy, J. Park, C. Chang, A. V. Soldatov, S. Lebedkin, R. C. Bialczak, J. E. Grose, L. A. K. Donev, J. P. Sethna, D. C. Ralph, and P. L. McEuen, Nano Lett. 5, 203 (2005).

[10] W. H. A. Thijssen, D. Djukic, A. F. Otte, R. H. Bremmer, and J. M. van Ruitenbeek, Phys. Rev. Lett. 97, 226806 (2006).

[11] T. Böhler, A. Edtbauer, and E. Scheer, Phys. Rev. B 76, 125432 (2007).

[12] N. P. de Leon, W. Liang, Q. Gu, and H. Park, Nano Lett. 8, 2963 (2008).

[13] K. J. Franke and J. I. Pascual, J. Phys.: Condens. Matter 24, 394002 (2012).

[14] J. Nygård and D. H. Cobden, Appl. Phys. Lett. 79, 4216 (2001).

[15] B. J. LeRoy, S. G. Lemay, J. Kong, and C. Dekker, Nature (London) 432, 371 (2004).

[16] S. Sapmaz, P. Jarillo-Herrero, Y. M. Blanter, and H. S. J. van der Zant, New J. Phys. 7, 243 (2005).

[17] B. J. LeRoy, J. Kong, V. K. Pahilwani, C. Dekker, and S. G. Lemay, Phys. Rev. B 72, 075413 (2005).

[18] S. Sapmaz, P. Jarillo-Herrero, Y. M. Blanter, C. Dekker, and H. S. J. van der Zant, Phys. Rev. Lett. 96, 026801 (2006).

[19] R. Leturcq, C. Stampfer, K. Inderbitzin, L. Durrer, C. Hierold, E. Mariani, M. G. Schultz, F. von Oppen, and K. Ensslin, Nat. Phys. 5, 327 (2009).

[20] A. K. Hüttel, B. Witkamp, M. Leijnse, M. R. Wegewijs, and H. S. J. van der Zant, Phys. Rev. Lett. 102, 225501 (2009).

[21] G. A. Steele, A. K. Hüttel, B. Witkamp, M. Poot, H. B. Meerwaldt, L. P. Kouwenhoven, and H. S. J. van der Zant, Science 325, 1103 (2009).

[22] B. Lassagne, Y. Tarakanov, J. Kinaret, D. Garcia-Sanchez, and A. Bachtold, Science 325, 1107 (2009).

[23] P. Utko, R. Ferone, I. V. Krive, R. I. Shekhter, M. Jonson, M. Monthioux, L. Noé, and J. Nygård, Nat. Commun. 1, 37 (2010).

[24] J. O. Island, V. Tayari, S. Yiğen, A. C. McRae, and A. R. Champagne, Appl. Phys. Lett. 99, 243106 (2011).

[25] M. Ganzhorn and W. Wernsdorfer, Phys. Rev. Lett. 108, 175502 (2012).

[26] D. R. Schmid, P. L. Stiller, C. Strunk, and A. K. Hüttel, New J. Phys. 14, 083024 (2012).

[27] H. B. Meerwaldt, G. Labadze, B. H. Schneider, A. Taspinar, Y. M. Blanter, H. S. J. van der Zant, and G. A. Steele, Phys. Rev. B 86, 115454 (2012).

[28] J. O. Island, V. Tayari, A. C. McRae, and A. R. Champagne, Nano Lett. 12, 4564 (2012).

[29] A. Benyamini, A. Hamo, S. V. Kusminskiy, F. von Oppen, and S. Ilani, Nat. Phys. 10, 151 (2014).

[30] P. Weber, H. L. Calvo, J. Bohle, K. Goß, C. Meyer, M. R. Wegewijs, and C. Stampfer, Nano Lett. 15, 4417 (2015).

[31] G.-W. Deng, D. Zhu, X.-H. Wang, C.-L. Zou, J.-T. Wang, H.-O. Li, G. Cao, D. Liu, Y. Li, M. Xiao, G.-C. Guo, K.-L. Jiang, X.-C. Dai, and G.-P. Guo, Nano Lett. 16, 5456 (2016).

[32] M. P. Blencowe, Contemp. Phys. 46, 249 (2005).

[33] Y. S. Greenberg, Y. A. Pashkin, and E. Il'ichev, Uspekhi. Fizicheskikh Nauk 182, 407 (2012).
[34] M. Poot and H. S. J. van der Zant, Phys. Rep. 511, 273 (2012).

[35] R. G. Knobel and A. N. Cleland, Nature (London) 424, 291 (2003).

[36] A. D. Armour, M. P. Blencowe, and Y. Zhang, Phys. Rev. B 69, 125313 (2004).

[37] Y. M. Blanter, O. Usmani, and Y. V. Nazarov, Phys. Rev. Lett. 93, 136802 (2004).

[38] N. M. Chtchelkatchev, W. Belzig, and C. Bruder, Phys. Rev. B 70, 193305 (2004).

[39] C. B. Doiron, W. Belzig, and C. Bruder, Phys. Rev. B 74, 205336 (2006).

[40] M. D. LaHaye, Science 304, 74 (2004).

[41] A. Naik, O. Buu, M. D. LaHaye, A. D. Armour, A. A. Clerk, M. P. Blencowe, and K. C. Schwab, Nature (London) 443, 193 (2006).

[42] A. A. Clerk and S. Bennett, New J. Phys. 7, 238 (2005).

[43] M. P. Blencowe, J. Imbers, and A. D. Armour, New J. Phys. 7, 236 (2005).

[44] V. Koerting, T. L. Schmidt, C. B. Doiron, B. Trauzettel, and C. Bruder, Phys. Rev. B 79, 134511 (2009).

[45] G. S. Paraoanu and A. M. Halvari, Appl. Phys. Lett. 86, 093101 (2005).

[46] A. Erbe, C. Weiss, W. Zwerger, and R. H. Blick, Phys. Rev. Lett. 87, 096106 (2001).

[47] L. Y. Gorelik, A. Isacsson, Y. M. Galperin, R. I. Shekhter, and M. Jonson, Nature (London) 411, 454 (2001).

[48] T. Novotný, A. Donarini, and A.-P. Jauho, Phys. Rev. Lett. 90, 256801 (2003).

[49] D. Fedorets, Phys. Rev. B 68, 033106 (2003).

[50] L. Y. Gorelik, S. I. Kulinich, R. I. Shekhter, M. Jonson, and V. M. Vinokur, in Theory of Quantum Transport in Metallic and Hybrid Nanostructures (Springer, Netherlands, Dordrecht, 2006), pp. 307-319.

[51] A. Y. Smirnov, L. G. Mourokh, and N. J. M. Horing, Phys. Rev. B 69, 155310 (2004).

[52] D. Fedorets, L. Y. Gorelik, R. I. Shekhter, and M. Jonson, Phys. Rev. Lett. 92, 166801 (2004).

[53] F. Pistolesi, Phys. Rev. B 69, 245409 (2004).

[54] A. Isacsson and T. Nord, Europhys. Lett. 66, 708 (2004).

[55] C. Kim, M. Prada, G. Platero, and R. H. Blick, Phys. Rev. Lett. 111, 197202 (2013).

[56] M. Prada, G. Platero, and D. Pfannkuche, Phys. Rev. B 89, 045426 (2014).

[57] E. M. Weig, R. H. Blick, T. Brandes, J. Kirschbaum, W. Wegscheider, M. Bichler, and J. P. Kotthaus, Phys. Rev. Lett. 92, 046804 (2004).

[58] Y. Okazaki, I. Mahboob, K. Onomitsu, S. Sasaki, and H. Yamaguchi, Nat. Commun. 7, 11132 (2016).

[59] M. Poot, S. Etaki, I. Mahboob, K. Onomitsu, H. Yamaguchi, Y. M. Blanter, and H. S. J. van der Zant, Phys. Rev. Lett. 105, 207203 (2010).

[60] M. Galperin, M. A. Ratner, and A. Nitzan, Nano Lett. 5, 125 (2005).

[61] D. Mozyrsky, M. B. Hastings, and I. Martin, Phys. Rev. B 73, 035104 (2006).

[62] F. Pistolesi and S. Labarthe, Phys. Rev. B 76, 165317 (2007).

[63] F. Pistolesi, Y. M. Blanter, and I. Martin, Phys. Rev. B 78, 085127 (2008). 
[64] G. Micchi, R. Avriller, and F. Pistolesi, Phys. Rev. Lett. 115, 206802 (2015).

[65] G. Micchi, R. Avriller, and F. Pistolesi, Phys. Rev. B 94, 125417 (2016).

[66] O. Usmani, Y. M. Blanter, and Y. V. Nazarov, Phys. Rev. B 75, 195312 (2007).

[67] G. Sonne, R. I. Shekhter, L. Y. Gorelik, S. I. Kulinich, and M. Jonson, Phys. Rev. B 78, 144501 (2008).

[68] D. Radić, A. Nordenfelt, A. M. Kadigrobov, R. I. Shekhter, M. Jonson, and L. Y. Gorelik, Phys. Rev. Lett. 107, 236802 (2011).

[69] J. Atalaya and L. Y. Gorelik, Phys. Rev. B 85, 245309 (2012).

[70] A. Vikstr om, A. M. Eriksson, S. I. Kulinich, and L. Y. Gorelik, Phys. Rev. Lett. 117, 247701 (2016).

[71] A. Nocera, C. A. Perroni, V. Marigliano Ramaglia, and V. Cataudella, Phys. Rev. B 86, 035420 (2012).

[72] A. Nocera, C. A. Perroni, V. M. Ramaglia, G. Cantele, and V. Cataudella, Phys. Rev. B 87, 155435 (2013).

[73] L. I. Glazman and R. I. Shekhter, Sov. Phys. JETP 67, 163 (1988).

[74] N. S. Wingreen, K. W. Jacobsen, and J. W. Wilkins, Phys. Rev. B 40, 11834 (1989).

[75] J. König, H. Schoeller, and G. Schön, Phys. Rev. Lett. 76, 1715 (1996).

[76] D. Boese and H. Schoeller, Europhys. Lett. 54, 668 (2001).

[77] U. Lundin and R. H. McKenzie, Phys. Rev. B 66, 075303 (2002).

[78] D. M.-T. Kuo and Y. C. Chang, Phys. Rev. B 66, 085311 (2002).

[79] K. D. McCarthy, N. Prokof'ev, and M. T. Tuominen, Phys. Rev. B 67, 245415 (2003).

[80] S. Braig and K. Flensberg, Phys. Rev. B 68, 205324 (2003).

[81] K. Flensberg, Phys. Rev. B 68, 205323 (2003).

[82] M. Galperin, M. A. Ratner, and A. Nitzan, J. Chem. Phys. 121, 11965 (2004).

[83] A. Mitra, I. Aleiner, and A. J. Millis, Phys. Rev. B 69, 245302 (2004).

[84] M. R. Wegewijs and K. C. Nowack, New J. Phys. 7, 239 (2005).

[85] G. A. Kaat and K. Flensberg, Phys. Rev. B 71, 155408 (2005).

[86] J. Koch and F. von Oppen, Phys. Rev. Lett. 94, 206804 (2005).

[87] J. Koch and F. von Oppen, Phys. Rev. B 72, 113308 (2005).

[88] A. Zazunov, D. Feinberg, and T. Martin, Phys. Rev. B 73, 115405 (2006).

[89] J. Koch, M. Semmelhack, F. von Oppen, and A. Nitzan, Phys. Rev. B 73, 155306 (2006).

[90] J. Koch, F. von Oppen, and A. V. Andreev, Phys. Rev. B 74, 205438 (2006)

[91] M.-J. Hwang, M.-S. Choi, and R. López, Phys. Rev. B 76, 165312 (2007).

[92] M. C. Lüffe, J. Koch, and F. von Oppen, Phys. Rev. B 77, 125306 (2008).

[93] M. Tahir and A. MacKinnon, Phys. Rev. B 77, 224305 (2008).

[94] O. Entin-Wohlman, Y. Imry, and A. Aharony, Phys. Rev. B 80, 035417 (2009).

[95] F. Haupt, T. Novotný, and W. Belzig, Phys. Rev. Lett. 103, 136601 (2009).

[96] F. Haupt, T. Novotný, and W. Belzig, Phys. Rev. B 82, 165441 (2010).

[97] M. G. Schultz, Phys. Rev. B 82, 155408 (2010).

[98] F. Cavaliere, E. Mariani, R. Leturcq, C. Stampfer, and M. Sassetti, Phys. Rev. B 81, 201303 (2010).
[99] A. Yar, A. Donarini, S. Koller, and M. Grifoni, Phys. Rev. B 84, 115432 (2011).

[100] G. Piovano, F. Cavaliere, E. Paladino, and M. Sassetti, Phys. Rev. B 83, 245311 (2011).

[101] T. Novotný, F. Haupt, and W. Belzig, Phys. Rev. B 84, 113107 (2011).

[102] T.-F. Fang, Q.-f. Sun, and H.-G. Luo, Phys. Rev. B 84, 155417 (2011).

[103] Z.-Z. Li, S.-H. Ouyang, C.-H. Lam, and J. Q. You, Phys. Rev. B 85, 235420 (2012).

[104] G. A. Skorobagatko, Phys. Rev. B 85, 075310 (2012).

[105] G. Rastelli, M. Houzet, L. Glazman, and F. Pistolesi, C. R. Phys. 13, 410 (2012).

[106] D. H. Santamore, N. Lambert, and F. Nori, Phys. Rev. B 87, 075422 (2013).

[107] R. Härtle, M. Butzin, and M. Thoss, Phys. Rev. B 87, 085422 (2013).

[108] M. Knap, E. Arrigoni, and W. von der Linden, Phys. Rev. B 88, 054301 (2013).

[109] S. Walter, B. Trauzettel, and T. L. Schmidt, Phys. Rev. B 88, 195425 (2013).

[110] B. K. Agarwalla, J.-H. Jiang, and D. Segal, Phys. Rev. B 92, 245418 (2015).

[111] C. Schinabeck, A. Erpenbeck, R. Härtle, and M. Thoss, Phys. Rev. B 94, 201407 (2016).

[112] J. K. Sowa, J. A. Mol, G. A. D. Briggs, and E. M. Gauger, Phys. Rev. B 95, 085423 (2017).

[113] D. A. Ryndyk, M. Hartung, and G. Cuniberti, Phys. Rev. B 73, 045420 (2006).

[114] A. Zazunov and T. Martin, Phys. Rev. B 76, 033417 (2007).

[115] R. Egger and A. O. Gogolin, Phys. Rev. B 77, 113405 (2008).

[116] L. K. Dash, H. Ness, and R. W. Godby, J. Chem. Phys. 132, 104113 (2010).

[117] G. Rastelli, M. Houzet, and F. Pistolesi, Europhys. Lett. 89, 57003 (2010).

[118] L. K. Dash, H. Ness, and R. W. Godby, Phys. Rev. B 84, 085433 (2011).

[119] R. Seoane Souto, A. Levy Yeyati, A. Martín-Rodero, and R. C. Monreal, Phys. Rev. B 89, 085412 (2014).

[120] H.-T. Chen, G. Cohen, A. J. Millis, and D. R. Reichman, Phys. Rev. B 93, 174309 (2016).

[121] H. Mera, T. G. Pedersen, and B. K. Nikolić, Phys. Rev. Lett. 115, 143001 (2015).

[122] Y. Pavlyukh, Sci. Rep. 7, 601 (2017).

[123] L. Mühlbacher and E. Rabani, Phys. Rev. Lett. 100, 176403 (2008).

[124] R. Hützen, S. Weiss, M. Thorwart, and R. Egger, Phys. Rev. B 85, 121408 (2012).

[125] E. Eidelstein, D. Goberman, and A. Schiller, Phys. Rev. B 87, 075319 (2013).

[126] A. Jovchev and F. B. Anders, Phys. Rev. B 87, 195112 (2013).

[127] R. Seoane Souto, R. Avriller, R. C. Monreal, A. Martín-Rodero, and A. Levy Yeyati, Phys. Rev. B 92, 125435 (2015).

[128] T. Novotný, A. Rossini, and K. Flensberg, Phys. Rev. B 72, 224502 (2005).

[129] A. Zazunov, R. Egger, C. Mora, and T. Martin, Phys. Rev. B 73, 214501 (2006).

[130] A. Zazunov, D. Feinberg, and T. Martin, Phys. Rev. Lett. 97, 196801 (2006). 
[131] G. Sonne, M. E. Peña-Aza, L. Y. Gorelik, R. I. Shekhter, and M. Jonson, Phys. Rev. Lett. 104, 226802 (2010).

[132] G. Sonne, M. E. Peña-Aza, R. I. Shekhter, L. Y. Gorelik, and M. Jonson, Low Temp. Phys. 36, 902 (2010).

[133] I. A. Sadovskyy, G. B. Lesovik, T. Jonckheere, and T. Martin, Phys. Rev. B 82, 235310 (2010).

[134] C. Padurariu, C. J. H. Keijzers, and Y. V. Nazarov, Phys. Rev. B 86, 155448 (2012).

[135] B. H. Wu, J. C. Cao, and C. Timm, Phys. Rev. B 86, 035406 (2012).

[136] A. Marchenkov, Z. Dai, B. Donehoo, R. N. Barnett, and U. Landman, Nat. Nanotechnol. 2, 481 (2007).

[137] A. V. Kretinin, A. Das, and H. Shtrikman, arXiv:1303.1410.

[138] Q.-F. Sun, J. Wang, and T.-H. Lin, Phys. Rev. B 59, 13126 (1999).

[139] J. Baranski and T. Domanski, J. Phys.: Condens. Matter 27, 305302 (2015).

[140] S.-N. Zhang, W. Pei, T.-F. Fang, and Q.-f. Sun, Phys. Rev. B 86, 104513 (2012).

[141] Q. Wang, H. Xie, H. Jiao, and Y.-H. Nie, Europhys. Lett. 101, 47008 (2013).

[142] B. Dong, G. H. Ding, and X. L. Lei, Phys. Rev. B 95, 035409 (2017).

[143] J.-D. Pillet, P. Joyez, R. Žitko, and M. F. Goffman, Phys. Rev. B 88, 045101 (2013).

[144] J. Schindele, A. Baumgartner, R. Maurand, M. Weiss, and C. Schönenberger, Phys. Rev. B 89, 045422 (2014).

[145] J. Gramich, A. Baumgartner, and C. Schönenberger, Phys. Rev. Lett. 115, 216801 (2015).

[146] J. Gramich, A. Baumgartner, and C. Schönenberger, Appl. Phys. Lett. 108, 172604 (2016).

[147] J. Gramich, A. Baumgartner, and C. Schönenberger, arXiv:1612.01201.

[148] P. Stadler, W. Belzig, and G. Rastelli, Phys. Rev. Lett. 117, 197202 (2016).

[149] U. Lundin, Phys. Lett. A 332, 127 (2004).

[150] F. Pistolesi, J. Low Temp. Phys. 154, 199 (2009).

[151] S. Zippilli, G. Morigi, and A. Bachtold, Phys. Rev. Lett. 102, 096804 (2009).

[152] S. Zippilli, A. Bachtold, and G. Morigi, Phys. Rev. B 81, 205408 (2010).

[153] Z.-Z. Li, S.-H. Ouyang, C.-H. Lam, and J. Q. You, Europhys. Lett. 95, 40003 (2011).

[154] F. Santandrea, L. Y. Gorelik, R. I. Shekhter, and M. Jonson, Phys. Rev. Lett. 106, 186803 (2011).

[155] L. Arrachea, N. Bode, and F. von Oppen, Phys. Rev. B 90, 125450 (2014).
[156] P. Stadler, W. Belzig, and G. Rastelli, Phys. Rev. Lett. 113, 047201 (2014).

[157] P. Stadler, W. Belzig, and G. Rastelli, Phys. Rev. B 91, 085432 (2015).

[158] P. J. Koppinen and I. J. Maasilta, Phys. Rev. Lett. 102, 165502 (2009).

[159] J. P. Pekola and F. W. J. Hekking, Phys. Rev. Lett. 98, 210604 (2007).

[160] R. Fazio and R. Raimondi, Phys. Rev. Lett. 80, 2913 (1998).

[161] P. Schwab and R. Raimondi, Phys. Rev. B 59, 1637 (1999).

[162] A. A. Clerk, V. Ambegaokar, and S. Hershfield, Phys. Rev. B 61, 3555 (2000).

[163] J. C. Cuevas, A. Levy Yeyati, and A. Martín-Rodero, Phys. Rev. B 63, 094515 (2001).

[164] Y. Tanaka, N. Kawakami, and A. Oguri, J. Phys. Soc. Jpn. 76 074701 (2007).

[165] A. Martín-Rodero and A. Levy Yeyati, Adv. Phys. 60, 899 (2011).

[166] T. Meng, S. Florens, and P. Simon, Phys. Rev. B 79, 224521 (2009).

[167] S. Droste, S. Andergassen, and J. Splettstoesser, J. Phys.: Condens. Matter 24, 415301 (2012).

[168] A. Braggio, M. Governale, M. G. Pala, and J. König, Solid State Commun. 151, 155 (2011).

[169] S. Droste, J. Splettstoesser, and M. Governale, Phys. Rev. B 91, 125401 (2015).

[170] R. S. Deacon, Y. Tanaka, A. Oiwa, R. Sakano, K. Yoshida, K. Shibata, K. Hirakawa, and S. Tarucha, Phys. Rev. Lett. 104, 076805 (2010).

[171] J. C. Cuevas, A. Martín-Rodero, and A. Levy Yeyati, Phys. Rev. B 54, 7366 (1996).

[172] G. E. Blonder, M. Tinkham, and T. M. Klapwijk, Phys. Rev. B 25, 4515 (1982).

[173] J. Rammer, Quantum Field Theory of Nonequilibrium States, 1st ed. (Cambridge University Press, New York, 2007).

[174] A. A. Clerk, Phys. Rev. B 70, 245306 (2004).

[175] Notice that even if we formally calculated the current in the normal lead $\hat{I}_{N}$, the expression for the inelastic current Eq. (47) is independent of this assumption, see further discussions in Refs. [176-179].

[176] J. K. Viljas, J. C. Cuevas, F. Pauly, and M. Häfner, Phys. Rev. B 72, 245415 (2005).

[177] M. Paulsson, T. Frederiksen, and M. Brandbyge, Phys. Rev. B 72, 201101 (2005).

[178] K. S. Thygesen and A. Rubio, Phys. Rev. B 77, 115333 (2008).

[179] H. Mera, M. Lannoo, C. Li, N. Cavassilas, and M. Bescond, Phys. Rev. B 86, 161404 (2012). 